\title{
The Substrate Import Mechanism of the Human Serotonin Transporter
}

\author{
Matthew C. Chan, ${ }^{\dagger, \triangle}$ Balaji Selvam, ${ }^{\dagger, \triangle}$ Heather J. Young, ${ }^{\ddagger}, \triangle$ Jihye Park, ${ }^{\ddagger}$ Erik \\ Procko, ${ }^{*,+,}, \boldsymbol{\Phi}, \S, \|$ and Diwakar Shukla*,†, $,, \|, \perp, \#, @$ \\ $\dagger$ Department of Chemical and Biomolecular Engineering, University of Illinois at \\ Urbana-Champaign, Urbana, IL, 61801 \\ $\ddagger$ Department of Biochemistry, University of Illinois at Urbana-Champaign, Urbana, IL,
} 61801

【enter for Biophysics and Quantitative Biology, University of Illinois at Urbana-Champaign, Urbana, IL, 61801

$\S$ Neuroscience Program, University of Illinois at Urbana-Champaign, Urbana, IL, 61801 \| Cancer Center at Illinois, University of Illinois at Urbana-Champaign, Urbana, IL, 61801 $\perp$ National Center for Supercomputing Applications, University of Illinois, Urbana, IL, 61801

\#Beckman Institute for Advanced Science and Technology, University of Illinois at Urbana-Champaign, Urbana, IL, 61801

@ NIH Center for Macromolecular Modeling and Bioinformatics, University of Illinois at Urbana-Champaign, Urbana, IL, 61801

$\triangle$ These authors contributed equally.

E-mail: procko@illinois.edu; diwakar@illinois.edu

\begin{abstract}
The serotonin transporter, SERT, initiates the reuptake of extracellular serotonin
\end{abstract}


in the synapse to terminate neurotransmission. Recently, the cryo-EM structures of SERT bound to ibogaine resolved in different states provided a glimpse of functional conformations at atomistic resolution. However, the conformational dynamics and structural transitions to various intermediate states are not fully understood. Furthermore, while experimental SERT structures were complexed with drug molecules and inhibitors, the molecular basis of how the physiological substrate, serotonin, is recognized, bound, and transported remains unclear. In this study, we performed microsecond long simulations of the human SERT to investigate the structural dynamics to various intermediate states and elucidated the complete substrate import pathway. Using Markov state models, we characterized a sequential order of conformational driven ion-coupled substrate binding and transport events and calculated the free energy barriers of conformation transitions associated with the import mechanism. We identified a set of residues that recognize the substrate at the extracellular surface of SERT and our simulations also revealed a third sodium ion binding site coordinated by Glu136 and Glu508 in a buried cavity which helps maintain the conserved fold adjacent to the orthosteric site for transport function. The mutation of these residues results in a complete loss of transport activity. Our study provides novel insights on the molecular basis of dynamics driven ion-substrate recognition and transport of SERT that can serve as a model for other closely related neurotransmitter transporters.

\section{Introduction}

The serotonin transporter (SERT) terminates synaptic transmission by catalyzing the reuptake of extracellular serotonin from the synapse. Reuptake is critical for normal serotonergic signaling in the brain with implications on mood, cognition, behavior, and appetite. ${ }^{1}$ Consequently, improper SERT function is associated with numerous neurological disorders including depression, autism, and bipolar disorder. ${ }^{2}$ Additionally, SERT is expressed in platelet membranes and regulates blood coagulation throughout the circulatory system. ${ }^{3}$ Given its 
medical importance, SERT is a major molecular target for therapeutic drugs and drugs of abuse. ${ }^{4,5}$ Similar to other members of the solute carrier 6 (SLC6) neurotransmitter transporter family, SERT mediated serotonin (5-hydroxytryptamine; 5HT) translocation from the synapse and surrounding area is coupled to favorable ion co-transport of one $\mathrm{Na}^{+}$with a $\mathrm{Cl}^{-}$ ion dependence, and export of one $\mathrm{K}^{+}$to complete an overall electroneutral cycle. ${ }^{6-10}$ Other conduction states and stoichiometries with unclear physiological significance may occur under different conditions. ${ }^{11-15}$

SERT, and the closely related dopamine transporter (DAT) and norepinephrine transporter (NET), belongs to a class of monoamine transporters in the neurotransmitter:sodium symporter (NSS) family. These members share a distinct 12 transmembrane (TM) helix architecture known as the LeuT fold, which consist of 12 transmembrane (TM) helices, with TM1-5 and TM6-10 forming inverted pentahelical repeats around a pseudo two-fold axis of symmetry. ${ }^{16,17}$ Cysteine labeling studies on SERT revealed that the 5HT binding site is accessible from both extracellular and intracellular sides of the membrane, providing the first glimpse of evidence of an alternating access model. ${ }^{18}$ Quick and Javitch developed a proteomic approach to characterize the sodium-dependent substrate transport mechanism in the tyrosine transporter Tyt $1 .{ }^{19}$ These biochemical studies elucidated that the NSS family of transporters function based on the principle of an alternating access mechanism. ${ }^{20}$ Crystal structures of the bacterial NSS homolog leucine transporter (LeuT) obtained in three functional states, outward-facing (OF), occluded (OC), and inward-facing (IF) states, have validated the NSS transport process is by an alternating access mechanism, in which the substrate and ions first access their central binding sites via an open extracellular vestibule, and then are released within the cell through the sequential closure of an extracellular gate and opening of an intracellular exit pathway. ${ }^{17,20-23}$ Historically, LeuT has served as a structural template to study monoamine transporters ${ }^{24-28}$ and based on in-depth studies of bacterial transporters, including electron paramagnetic resonance (EPR) spectroscopy ${ }^{29-31}$ molecular modeling, ${ }^{32,33}$ and single-molecule fluorescence resonance energy transfer (smFRET) 
experiments, ${ }^{28,34}$ substrate permeation through the NSS family transporters is facilitated by reorientation of helices around the central axis, in particular the movement of TM1a away from the helical bundle to open an intracellular vestibule for substrate release. ${ }^{21,35,36}$ Despite low sequence similarity with human NSS transporters, these efforts paved the way for rational drug design for treating various psychiatric disorders. ${ }^{37-39}$

Structural investigations into human NSS transporters have further benefited from the more recent resolution of outward-facing conformations of eukaryotic monoamine transporters Drosophila DAT ( $d$ DAT) and human SERT (hSERT). ${ }^{40-43}$ The screening and docking studies using these crystal structures provide the structural basis of antidepressant recognition and inhibition. ${ }^{44-49}$ Most recently, cryogenic electron microscopy (cryo-EM) structures of $h$ SERT complexed with the psychedelic non-competitive inhibitor ibogaine reveal the occluded and inward-facing states with similar structural arrangements as seen in LeuT. ${ }^{17,23,50,51}$ However, given the structural discrepancies between SERT and other NSS structural models, the molecular basis of transitions between the intermediate states remains unknown. Closure of the extracellular vestibule is coordinated by helix motions of TM1b and TM6a where Arg79 and Glu493 are proposed to serve as extracellular gating residues to stabilize the OC and IF states. The helix orientation of TM1b in the SERT OC conformation is closely aligned to that of OF LeuT. Moreover, among the current SERT OF structures, the distance between the guanidinium group of Arg79 and carboxyl of Glu493 varies from $4.4 \AA$ to $7.4 \AA$, while in the OC and IF states, this distance is $7.2 \AA$ and $4.6 \AA$, respectively. As a result, the role of these gating residues and their interactions during conformational transitions is unclear. The N-terminal loop preceding TM1a and its interactions with TM6 and TM8 regulates the helix motion of TM1a during substrate release and acts as an intracellular gate. ${ }^{36,52,53}$ Hydrogen-deuterium exchange (HDX) experiments have provided an alternative approach to understand the conformational dynamics within the NSS family and have shown that ion-substrate binding facilitates changes in dynamics in TM1a, TM6, and TM7. ${ }^{24,54-56}$ Intricate loop dynamics, specifically motions of extracellular loops (EL) 3 and 4 
fluctuates significantly during substrate transport. ${ }^{24,54-56}$ The combined structural and biochemical studies have provided invaluable insights in the functional mechanism of the NSS family. However, the realistic motions of structural transitions at atomistic resolution are not fully known to understand the conformational driven substrate transport cycle.

In this study, we performed unbiased all-atom molecular dynamics (MD) simulations to obtain a comprehensive understanding of the import mechanism for the physiological substrate serotonin in $h$ SERT. Our study shows the key binding and transport events, including substrate interactions at an extracellular allosteric site, neurotransmitter binding within subsite $\mathrm{B}$, coordination of three metal ions, and a single symported sodium ion being displaced into the cytosol by the movement of serotonin into the exit pathway. Using a Markov state model (MSM)-based adaptive sampling approach to explore the conformational landscape, we report a sequential order of the ion-substrate binding and transport processes for any NSS family transporter. The free energy conformational landscape plots reveal that structural isomerization from OC to IF is a rate-limiting step for import that is facilitated by the presence of $5 \mathrm{HT}$ in the orthosteric site. We identified a third sodium ion binding site in a buried cavity close to the orthosteric site which helps maintain the fold for substrate transport. We determined the key residues that are involved in 5HT recognition, binding, and transport, and these residues show to have some role in transport using site-directed mutagenesis. Our results provide an in-depth perspective into the molecular recognition and transport of $5 \mathrm{HT}$ in SERT and may aid for the development of conformational selective inhibitors for the treatment of psychiatric disorders. 


\section{Results}

\section{Substrate binding decreases the free energy barrier for SERT con- formational transitions to the IF state.}

To understand the effects of substrate-induced protein dynamics, the entire import process of 5HT was studied using molecular dynamics (MD) simulations. A Markov state model (MSM)-based adaptive sampling approach was used to explore the entire accessible conformational space of the SERT. ${ }^{57-61}$ Simulations were initiated from the OF crystal structure of $h$ SERT (PDB: 5I73) and a total of $130 \mu$ s of 5HT-free SERT (referred to as $\mathrm{Na}^{+}-\mathrm{SERT}$ ) was obtained. $\mathrm{Na}^{+}$-bound SERT in an OF conformation obtained from $\mathrm{Na}^{+}$-SERT simulations, with the Na1 and Na2 sites occupied, was used to seed simulations of the $5 \mathrm{HT}$ import process (referred to as 5HT-SERT). $100 \mathrm{mM}$ 5HT was added to the simulation box (equivalent to $125 \mathrm{HT}$ molecules) and a total of $210 \mu$ s data were collected. All simulation data were used to construct an MSM, which parses the simulation data into kinetically relevant states and calculates the transition probabilities between the states (See Methods for additional details). MSM-weighted simulation data were projected onto a coordinate system defined by distances between extracellular and intracellular gating residues (Figure 1 and S1).

The conformational landscape plots reveal that despite the absence of $5 \mathrm{HT}$ binding, $\mathrm{Na}^{+}$SERT may undergo transitions from the OF state to the IF state (Figure 1A). Extracellular gating residues Arg104 (TM1b) and Glu493 (TM10) can separate to $10 \AA$, enlarging the extracellular permeation pathway. The equivalent charged residues in the bacterial transporter LeuT (Arg20 and Asp404) have been previously implicated in the gating mechanism. ${ }^{17,62}$ The OF states are stable, with a relative free energy of $\sim 1.5 \mathrm{kcal} / \mathrm{mol}$. The distance between gating residues Arg104-Glu493 decreases to $3 \AA$ and is associated with electrostatic interactions (Figure 1 and S2), forming OC conformations that are more stable than the OF state. Previous studies have shown that binding of $\mathrm{Na}^{+}$stabilizes the extracellular open conformation in SERT and related transporters, agreeing with the free energy landscapes 

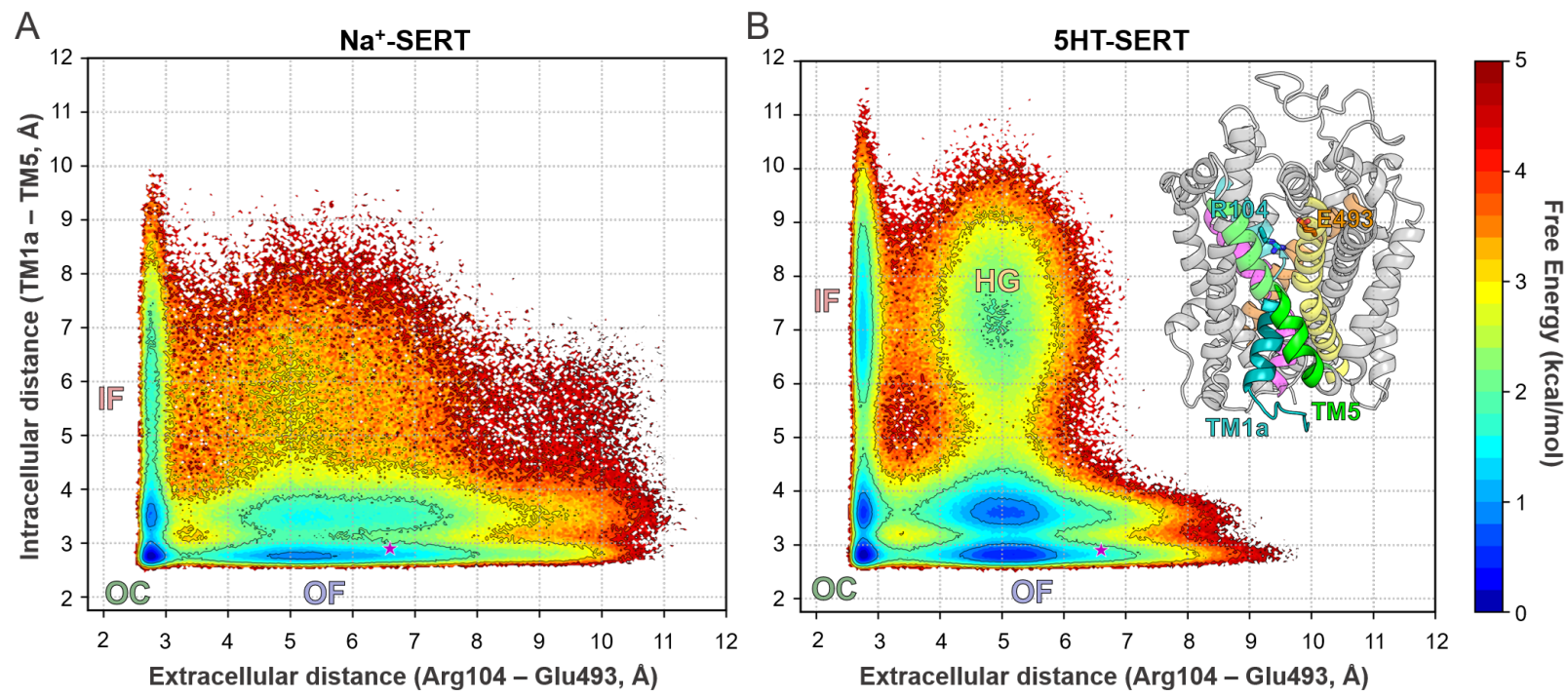

C

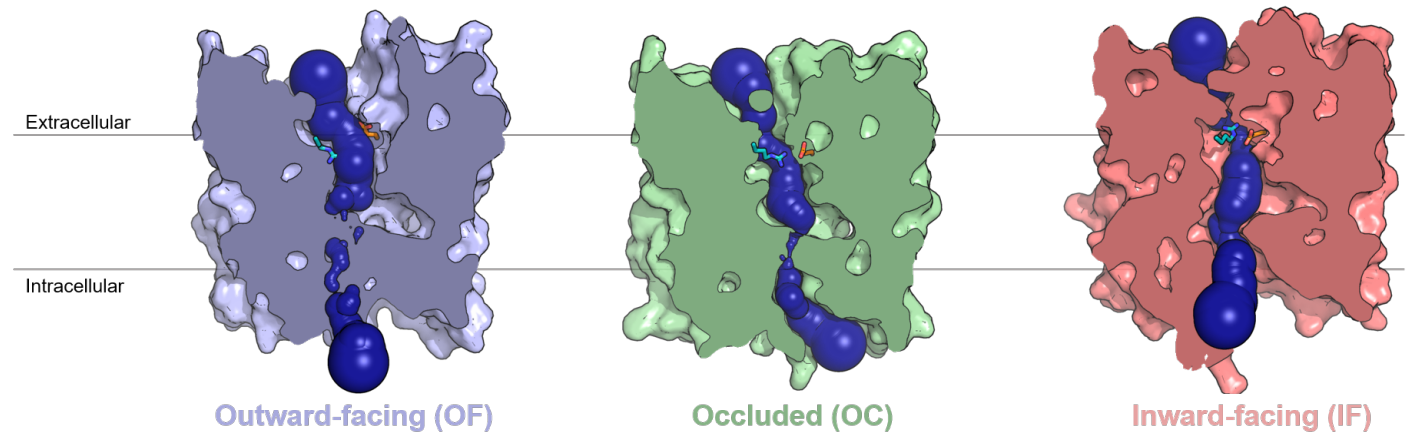

Figure 1: Conformational free energy landscapes of SERT obtained from MD simulations. Relative free energies from MSM-weighted simulation data plotted against the distances between extracellular and intracellular gates for (A) $\mathrm{Na}^{+}$-SERT and (B) 5HT-SERT. An outward-facing (OF) SERT crystal structure (PDB 5I73, pink star) was used as the starting structure for MD simulations and transitioned to occluded (OC) and inward-facing (IF) states. An hourglass-like (HG) state, in which both gates are open, was also observed. The SERT structure is represented as cartoon with TM 1, 5, 6, 8, and 10 colored in teal, green, magenta, yellow, and orange respectively. (C) Cross-section through SERT conformational states viewed from the membrane plane, shown as surface representations. The channel pore volume across the transporter is depicted as dark blue spheres and extracellular gates Arg104 and Glu493 are shown as teal and orange sticks, respectively. 
calculated from MD simulation. ${ }^{28,29,63-65}$ Distance distribution plots reveal that the extracellular surface remains open while the intracellular vestibule is closed in OF and OC states, and vice versa in the IF state (Figure S3). Closure of the extracellular pathway as SERT isomerizes from the OF to OC state weakens contacts on the intracellular side of the transporter, creating an energetically accessible pathway towards a partial IF state, in which the intracellular pathway measured by the opening of TM1a and TM5 extends to $\sim 6 \AA$. The free energy barrier for transition from the OC-IF state in $\mathrm{Na}^{+}$-SERT is estimated as $\sim 2$ $\mathrm{kcal} / \mathrm{mol}$, which is higher compared to the OF-OC transition $(\sim 1 \mathrm{kcal} / \mathrm{mol})$. Biophysical investigations of LeuT also reveal that IF states are less populated and that transitions to IF states are facilitated by the presence of the substrate. ${ }^{28,29,31,34}$ Formation of the IF state is associated with the partial unwinding of the cytoplasmic base of TM5, the breakage of electrostatic interactions between Arg79 (N-Term) and Asp452 (TM8) and Glu80 (N-Term) and Lys275 (TM5) at the intracellular gate, and increased dynamics of the flanking loops (Figure S2). Simulated helix rearrangements involved in opening and closing of the transporter agree with the recent cryo-EM structures ${ }^{50}$ and other NSS crystal structures ${ }^{23,44,46}$ (Figure S4).

The substrate-present conformational landscape plot exhibits deviations in the relative free energies of conformational states and reduced free energy barriers between states (Figure 1B). Binding of $5 \mathrm{HT}$ in the entrance pathway stabilizes the OF states to a greater extent compared to $\mathrm{Na}^{+}$-SERT simulations (Figure 1, S3C). ${ }^{28}$ The gating residues form alternative interactions with Gln332 (TM6) and Lys490 (TM10), thereby widening the extracellular vestibule (Figure S2). Similar to observations seen in human DAT simulations, ${ }^{40}$ the diffusion of $5 \mathrm{HT}$ to the orthosteric (S1) site via the allosteric (S2) site promotes the inward closure of extracellular gating helices TM1b, TM6a and TM10 to facilitate formation of the OC state (Figure S5). The OF-OC transition has a free energy barrier of $\sim 1.5 \mathrm{kcal} / \mathrm{mol}$, similar to $\mathrm{Na}^{+}$-SERT. The 'downward' movement of $5 \mathrm{HT}$ facilitates opening of the intracellular gate and isomerization to the IF state. The free energy barrier for the OC-IF structural 
transition is estimated as $\sim 2 \mathrm{kcal} / \mathrm{mol}$. The presence of $5 \mathrm{HT}$ in the intracellular pathway stabilizes SERT in a greater IF state, with a relative free energy of $\sim 1 \mathrm{kcal} / \mathrm{mol}$ as compared to $\sim 2 \mathrm{kcal} / \mathrm{mol}$ in $\mathrm{Na}^{+}-\mathrm{SERT}$. Compared to $\mathrm{Na}^{+}-\mathrm{SERT}$, the intracellular vestibule in the IF state now extends to 7-10 $\AA$, thus allowing for the substrate to be released and is associated with the breakage of hydrogen bonding networks between intracellular gating helices (Figure S2, S6, S7, S8, S9). Terry et al. has demonstrated that the selective substrate-driven conformational transition to the IF state in LeuT is highly favorable compared to nonselective substrates and ion binding alone. ${ }^{28}$ Using Transition path theory, we estimated the mean first passage time (MFPT) for different transitions observed in the simulations. We found that the rate of transition between OC-IF states is rate-limiting as compared to transitions between OF-OC states both in the presence and absence of 5HT (Figure S10). We also observed partial OF-IF like conformations, which we have termed as an hourglass-like (HG) state in which both gates are open but constricted at the center (Figure S11C). Terry et al. identified intermediate conformations in LeuT which are open at both extracellular and cytoplasmic halves and may enable the transport of $\mathrm{Na}^{+}$and substrate molecules. The conformational landscape plots reveal that these states are less stable $(>3 \mathrm{kcal} / \mathrm{mol})$ as compared to canonical intermediate states OF, OC and IF. Therefore, the release of $\mathrm{Na}^{+}$ ions and substrate molecules through HG states is a more energetically demanding process than the traditional substrate transport via the IF state. In $\mathrm{Na}^{+}$-SERT simulations, we observed uncoupled ion leaks, in contrast, $\mathrm{Na}^{+}$binds tightly to its respective site and no ion leaks were observed in 5HT-SERT (Figure S12). This state has been observed in other membrane transporters ${ }^{66-68}$ including a disease-associated mutant of DAT, ${ }^{69}$ but transitions from HG to other intermediate states are restricted as the free energy barriers are high and the physiological relevance of this state in SERT remains elusive.

The calculated root mean squared fluctuation (RMSF) plots illustrate that in both $\mathrm{Na}^{+}$ and 5HT-SERT simulations, EL2 and EL4 are highly flexible during the OF to OC transitions. Alternatively, the loop regions are more stable during OC to IF in 5HT-SERT simula- 


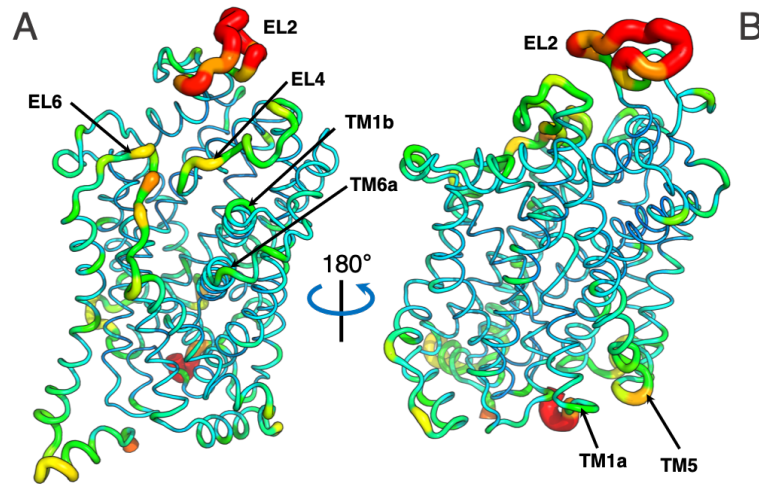

$\left(\mathrm{Na}^{+}-\mathrm{SERT}\right) \mathrm{OF} \rightarrow\left(\mathrm{Na}^{+}-\mathrm{SERT}\right) \mathrm{OC}$

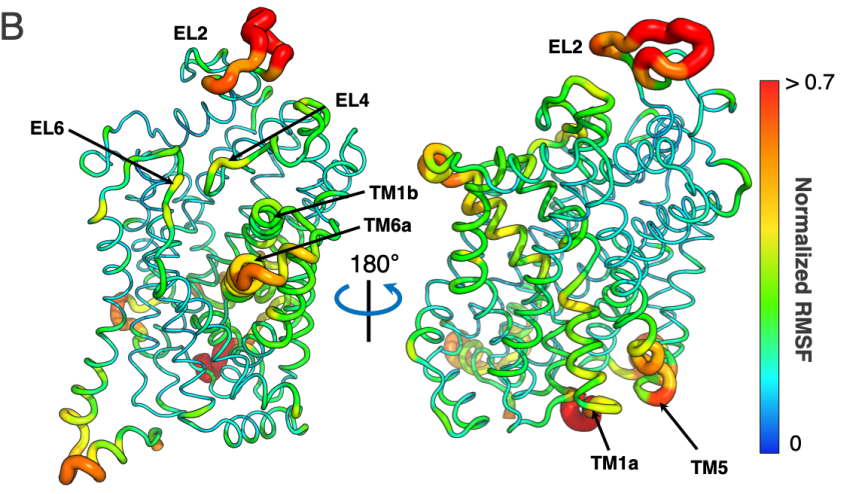

$\left(\mathrm{Na}^{+}-\mathrm{SERT}\right) \mathrm{OC} \rightarrow\left(\mathrm{Na}^{+}-\mathrm{SERT}\right)$ IF


Figure 2: Molecular dynamics analysis of global fluctuations during conformational transitions. Normalized RMSF of $\mathrm{Na}^{+}$(A,B) and 5HT transport (C,D) for OF to OC transition and OC to IF transition mapped to simulated SERT structure. Tube thickness corresponds to the RMSF values of each residue. 
tions compared to $\mathrm{Na}^{+}$simulations (Figure 2). Hydrogen deuterium exchange (HDX) mass spectroscopy studies hint that EL2 and EL4 regions are destabilized and show increased deuterium exchange upon ion and substrate binding. ${ }^{24,54,56,68}$ Furthermore, EL2 exhibits higher deuterium uptake kinetics in the OF state compared to the IF state in LeuT. ${ }^{24}$ In 5 HT-SERT simulations, EL4 shows less pronounced fluctuations during OC to IF transitions. The experimental results from HDX show EL4 regions are more stabilized during $\mathrm{K}^{+}$uptake which is hypothesized to stabilize the IF state of SERT based on LeuT studies. ${ }^{56,70}$ The increased deuterium uptake of TM1a in the presence of $\mathrm{K}^{+}$agrees with the large fluctuations we observed for transitions to the IF state. The comparison of calculated deuterium exchange fraction of $\mathrm{Na}^{+}$and 5HT-SERT simulation data agree with previous HDX studies (Figure S13). ${ }^{24}$

\section{Identification of a new sodium ion binding site in a buried cavity stabilizes the fold for substrate transport.}

Monoamine transporters utilize an electrochemical gradient to transport substrates across the cellular membrane. 5HT-mediated transport involves the symport of $1 \mathrm{Na}^{+}$ion and antiport of $1 \mathrm{~K}^{+}$ion. ${ }^{7,71}$ Upon the transition of $5 \mathrm{HT}$ from the allosteric to the orthosteric binding site, the $\mathrm{Na}^{+}$ion in the Na1 site shifts to a third metal coordination center, which we call the Na3 site to be consistent with prior nomenclature. ${ }^{72,73}$ The vacant Na1 site is then filled by two to three water molecules that interact with the charged site until another $\mathrm{Na}^{+}$ion binds to the Na1 site (Figure S14). The calculated electrostatic potential map of SERT OF state cryo-EM structure reveals that the permeation pathway is negatively charged, which allows $\mathrm{Na}^{+}$ions to rapidly diffuse and bind to the Na1 site (Figure S15).

At the Na3 site, the $\mathrm{Na}^{+}$ion is coordinated by the carboxylates of Glu136 and Glu508, and the sulfur of Met135 through water molecules (Figure 3). A third metal ion site has not previously been described in SERT, but computational modeling, biochemical analysis, and electrophysiology recordings indicate that equivalent residues of the neuronal GlyT2 
transporter also form a third $\mathrm{Na}^{+}$site. ${ }^{73}$ The simulation reveals that the presence of $\mathrm{Na}^{+}$ in the Na3 site stabilizes TM6 unwinding and the proper orientations of residues in the orthosteric site. There are two pieces of experimental evidence for this in silico discovery. First, a structural alignment of SERT from the MD simulation with the crystal structure shows weak but discernible electron density, comparable to the density of surrounding side chains, near the modeled third $\mathrm{Na}^{+}$(Figure 3). Second, previous experiments have shown a reduction of serotonin transport when Glu136 is mutated, underscoring the role of this residue for appropriate conformational dynamics. ${ }^{74}$

We note that the presence of buried glutamates within hydrophobic transmembrane regions is highly unusual; in this case, Glu136 hydrogen bonds to exposed backbone N-H groups to support the unwinding of TM6 near the central substrate binding site. We calculated the pKa of these residues with and without $\mathrm{Na} 3$ bound and found that they are not protonated under physiological conditions (Table S1). The calculated pKa values for SERT resolved structures and other NSS structures were also predicted to be in the range of 3-4, given the intracellular neuron $\mathrm{pH}$ is $7.17^{75}$ (Table S1). Our simulation shows that the Na3 cavity is well solvated before and after Na3 binding, with an average of 4-5 and 7-8 water molecules with $5 \AA$ of the Na3 site, respectively (Figure S16A, S16B). We also calculated the protein tunnels and channel-like pores that could facilitate the transport of small molecules and ions for the OF, OC and IF states of SERT. The tunnel plot reveals that the Na3 site is completely buried inside the transporter and is not exposed to the cytoplasmic half (Figure S16C). Therefore, from our simulations, we observed that Na3 is not released to the intracellular side (Figure S17E).

\section{Simulations reveal a sequential order of substrate binding and trans- port in SERT.}

An aspect of the current NSS transport model that remains unaddressed is the sequential order of substrate binding and transport events. Using transition path theory, the highest 


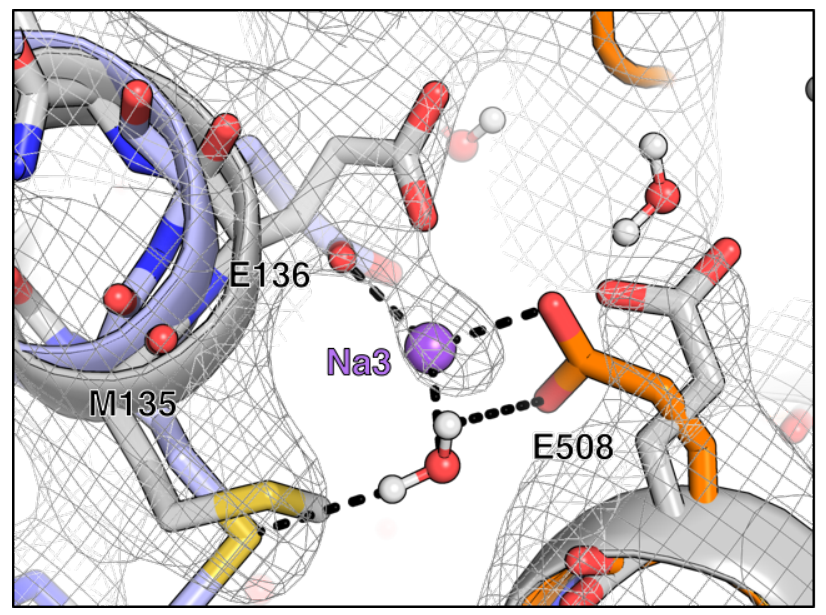

Figure 3: A third $\mathrm{Na}^{+}$ion binding site buried beneath the orthosteric pocket. MD snapshot (TM2 colored pale blue and TM10 orange) superimposed with the SERT crystal structure (PDB 5I73, grey), with electron density shown at $0.5 \sigma$.

flux pathway for conformational change and 5HT import can be determined from the Markov state model and used to predict an ordered sequence of binding events and structural changes. SERT undergoes complete transitions to the IF state in the simulations, with permeation towards the intracellular side upon binding of substrates in the order following $\mathrm{Na}^{+}, 5 \mathrm{HT}$, and $\mathrm{Cl}^{-}$ions (Figure 4). We describe each step of the import process in detail.

The transport process begins with the binding of $\mathrm{Na}^{+}$to the Na1 site, followed by a second $\mathrm{Na}^{+}$binding the Na2 site (Figure S18, S19). These two sites are well-described in the SERT crystal structure and the literature. ${ }^{23,44,46,76} \mathrm{Na}^{+}$bound at the Na1 site couples activity between the ion and substrate binding sites, whereas computational studies of related transporters have indicated that $\mathrm{Na}^{+}$coordinated at the $\mathrm{Na} 2$ site dissociates during the transporter cycle to become the symported metal ion. ${ }^{40,77,78}$ The importance of the Na2 site is underscored by its conservation in distantly related secondary transporters. ${ }^{79} \mathrm{Na}^{+}$ ions entering the transporter interact with Asp328 and Asn112 at the extracellular surface, then rapidly diffuse into the allosteric site (Figure S18A). Here, the $\mathrm{Na}^{+}$ions interact with Glu493 and Glu494, and a rotameric shift in Glu493 enables the ions to descend past the extracellular gate to their central binding sites (Figure S18B, S18C). Na1 is stabilized by 
(3)

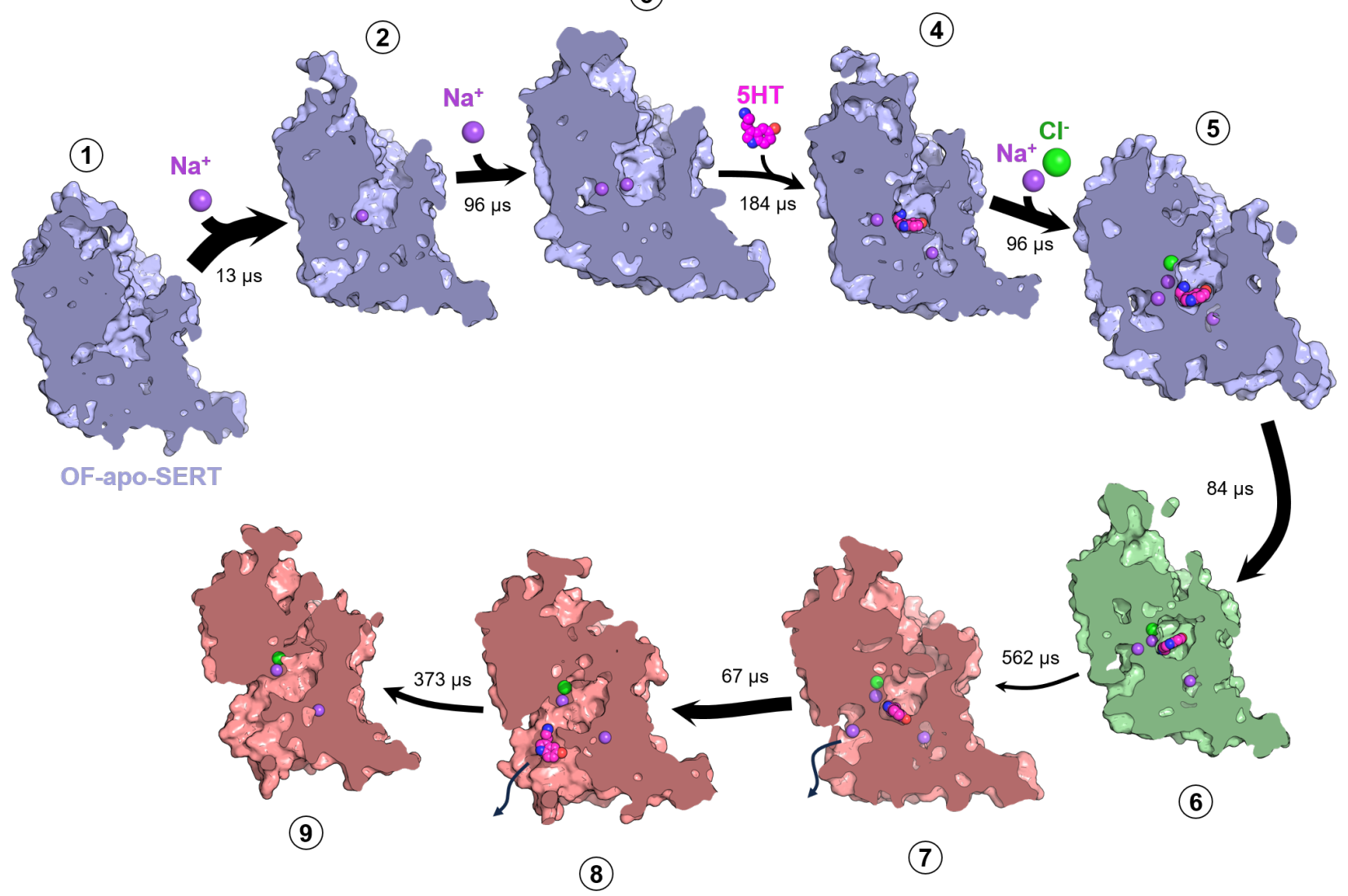

Figure 4: The major flux pathway and mean first passage times for SERT conformational transitions and 5HT import determined from transition path theory. The transport process begins with the binding of $2 \mathrm{Na}^{+}$ions to the Na1 and Na2 sites in the OF state $(2,3)$. Substrate diffusion to the orthosteric site shifts a $\mathrm{Na}^{+}$to the Na3 site (4). An additional $\mathrm{Na}^{+}$and $\mathrm{Cl}^{-}$ion bind (5), facilitating closure of the extracellular gate to form the OC state (6). Isomerization to the IF state is associated with the release of $\mathrm{Na}^{+}$from the Na2 site and 5HT diffuses out (6-9). Arrow thickness represents relative flux between transitions.

Asn101, Ser336, and Asn368, while Na2 is coordinated by backbone carbonyls of Gly94, Val97, Leu434, and side chains of Asp437 and Ser438. Previous simulations of SERT have shown Asp437 as a key residue for maintaining Na2-ion interactions, ${ }^{78}$ and mutations of Asp437 and Ser438 have confirmed their role in coordination and dissociation of Na2. ${ }^{78,80}$ Similar to other transporters in the NSS family, ${ }^{29,63,64}$ the binding of $\mathrm{Na}^{+}$ions to their respective sites stabilizes SERT in the OF state while neutralizing the polar cavity to allow protonated 5HT diffusion. 
5HT is recognized by Tyr107, Ile108, Gln111, and Asp328 at the extracellular vestibule to initiate the binding in the OF state. Ile108 forms hydrophobic contacts with the indole ring of $5 \mathrm{HT}$ while other residues form polar interactions with the substrate that favors binding. 5HT then diffuses inside the translocation pore and binds to the allosteric site (Figure 5B). The substrate is stabilized by aromatic ring packing against Phe335 and Arg104, and a hydrogen bonding network with Asp328, Gln332, and Glu494 (Figure 5B). Previous mutations of residues in the allosteric site have been shown to alter inhibitor potency. ${ }^{81} 5 \mathrm{HT}$ undergoes a $90^{\circ}$ rotation by rapidly exchanging its polar interactions and shifts towards the orthosteric site $5 \mathrm{C})$. The switching of amine group interactions to Glu494 triggers the movement of $5 \mathrm{HT}$ from the allosteric site to the orthosteric site. 5HT rotates such that the conformation becomes perpendicular to the membrane which is further favored by polar and hydrophobic interactions by Asp328, Gln332, Leu502, and Ala331. The extracellular gating residues form a salt bridge interaction and enlarges the binding cavity such that substrate can escape to the primary binding site. This substrate binding site in the NSS family contains three well-studies subsites and has served as the basis of designing various trycyclic antidepressant molecules. ${ }^{82}$ In the orthosteric site, the protonated amine moiety of 5HT forms charged interactions with Asp98 of subsite A, disrupting the hydrogen bonding interactions between Asp98 and Tyr176 (Figure S20). Also within subsite A, the phenol moiety forms aromatic interactions with Tyr95 and Phe341. Site-directed mutagenesis and computational docking studies have emphasized the significance of Asp98 in 5HT recognition and transport, ${ }^{83,84}$ while biochemical studies show that the disruption of aromatic interactions with the substrate leads to a loss of function or decreased potency of antidepressants. ${ }^{81,85-87}$ The binding of 5HT from the allosteric to the orthosteric binding site promotes $\mathrm{Na}^{+}$in the Na1 site to migrate to the third metal coordination center, the Na3 site. 

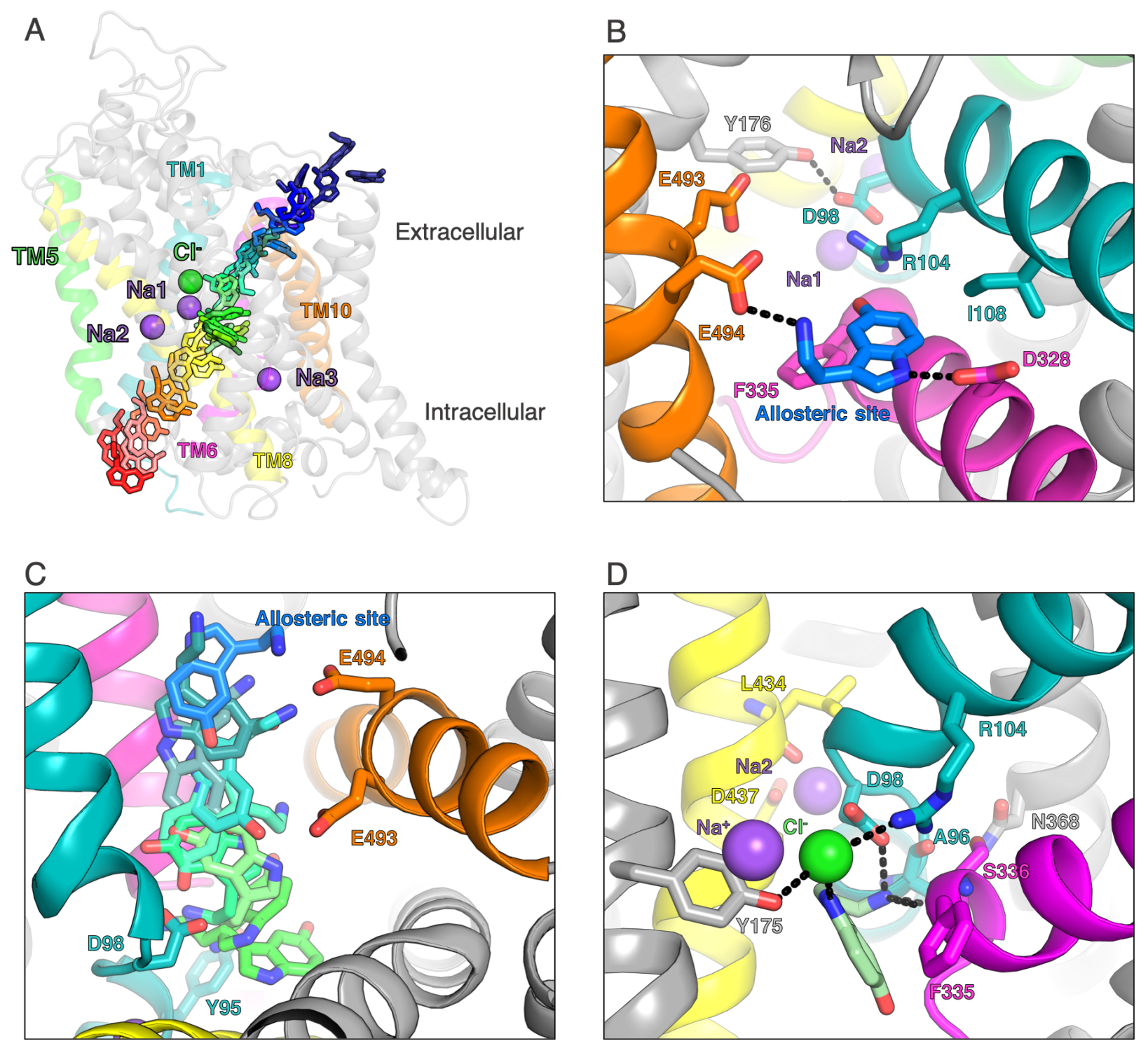

Figure 5: MD snapshots of the simulated mechanism for SERT-catalyzed 5HT import. (A) Overlaid MD snapshots of 5HT translocation, from when 5HT enters the extracellular vestibule (blue) to its cytosolic exit (red). The positions of ions in the OC state are shown as spheres. Transmembrane helices 1, 5, 6, 8, and 10, are colored in teal, green, magenta, yellow, and orange, respectively. (B) 5HT enters the transporter by binding in the allosteric site. (C) From the allosteric site, 5HT rotates down the transport pathway to the orthosteric site. (D) Initial $\mathrm{Cl}^{-}$recognition is assisted by Asp98, Arg104, Tyr175, Phe335, and indole of $5 \mathrm{HT}$.

\section{HT mediates the binding of an electrogenic $\mathrm{Cl}^{-}$ion.}

$\mathrm{Cl}^{-}$and $\mathrm{Na}^{+}$permeate into the extracellular vestibule and bind at the $\mathrm{Cl}^{-}$and vacated Na1 coordination sites, which leads to the formation of an occluded conformation (Figure S21). 
$\mathrm{Na}^{+}$is stabilized by Glu493 and Glu494 while $\mathrm{Cl}^{-}$forms polar interactions with Arg104 and Tyr176. The calculated electrostatic potential map of the SERT-OF cryo-EM structure reveals that the permeation pathway is negatively charged and supports our finding that the binding of $\mathrm{Cl}^{-}$ion will be accompanied by a $\mathrm{Na}^{+}$ion (Figure S15). The additional interaction of $\mathrm{Cl}^{-}$with the indole- $\mathrm{NH}$ of $5 \mathrm{HT}$ further stabilizes the ion in the exposed extracellular recognition site (Figure 5D). The indole ring of $5 \mathrm{HT}$ occupies subsite $\mathrm{C}$ of the primary binding site, which in turn favors the transition of the $\mathrm{Cl}^{-}$ion to its binding site (Figure $\mathrm{S} 22)$.

As $\mathrm{Cl}^{-}$enters, it shifts the guanidinium group of Arg104, facilitating the diffusion of both the $\mathrm{Cl}^{-}$and $\mathrm{Na}^{+}$ions into the central cavity (Figure S20C). The movement of $\mathrm{Cl}^{-}$ through the transporter is supported by a network of interactions with Arg104, Tyr176, and the indole-NH of 5HT (Figure 5D). A shift of the Arg104 side chain exposes Gln332 for making contacts with the $\mathrm{Cl}^{-}$ion, facilitating its migration to the $\mathrm{Cl}^{-}$binding site. The predicted Na1, Na2, and $\mathrm{Cl}^{-}$sites in the simulations concur with their respective sites observed in crystal structures of SERT, LeuT, and DAT ${ }^{23,44,46,76}$ (Figure S23). Interestingly, we did not observe $\mathrm{Cl}^{-}$to bind in the absence of the substrate within the given simulation timescales (Figure S24). In order to quantify the binding of $\mathrm{Cl}^{-}$in the absence of 5HT, we performed umbrella sampling simulations to compute the free energy of $\mathrm{Cl}^{-}$binding in $\mathrm{Na}^{+}$-SERT conditions. The free energy barrier for $\mathrm{Cl}^{-}$binding without $5 \mathrm{HT}$ was estimated on the order of $\sim 12 \mathrm{kcal} / \mathrm{mol}$ as compared to $\sim 4 \mathrm{kcal} / \mathrm{mol}$ in 5 HT-SERT simulations (Figure S25). The highest energy barrier observed at approximately $3 \AA$ away from the $\mathrm{Cl}^{-}$binding site is associated with the dissociation of $\mathrm{Na}^{+}$from $\mathrm{Cl}^{-}$to allow binding to their respective sites suggesting that the substrate in the orthosteric binding site neutralizes the polar cavity to allow for favorable $\mathrm{Cl}^{-}$binding.

The binding of ions and the substrate to their respective sites leads to the closure of the extracellular gates to obtain the OC state where the permeation pathway is closed at both ends. The decrease of the pore results in shifting of the aromatic ring of $5 \mathrm{HT}$ from subsite $\mathrm{C}$ 
to subsite $\mathrm{B}$ within the orthosteric binding pocket. The amine moiety of the neurotransmitter remains bound to Asp98, Tyr95, and the C-terminal pole of TM1a within subsite A (Figure $6 \mathrm{~A}, \mathrm{~B})$. The simulated configuration of $5 \mathrm{HT}$ in subsite $\mathrm{B}$ agrees with the crystal structure of dopamine-bound $d \mathrm{DAT}^{76}$ (Figure S23F). The indole-NH ring of 5HT interacts with Thr473 and other hydrophobic contacts by Ala169, Ala441, Gly442, and Leu443 residues stabilizing the substrate in subsite B. Additionally, 5HT may enter into subsite B in which the indole$\mathrm{NH}$ is oriented towards Phe341 adopting a binding pose also reported in previous modeling studies. ${ }^{84,88}$ Previous experimental studies indicate that mutations of these residues can decrease $5 \mathrm{HT}$ transport ${ }^{81,85}$ and the binding pose is further supported by several studies showing that interactions in subsite B are critical for inhibitor potency. ${ }^{82,85,86}$

\section{Mechanism of 5HT translocation down the exit pathway.}

The conformational free energy landscape suggests that structural isomerization to the IF state is limited by a large free energy barrier which is decreased in the presence of the substrate. The prolonged binding of $5 \mathrm{HT}$ in subsite $\mathrm{B}$ weakens $\mathrm{Na}^{+}$interactions in the $\mathrm{Na} 2$ site and results in its dissociation. $\mathrm{Na}^{+}$loses its interaction with the backbone carbonyl of Leu434 and enters the intracellular vestibule, thereby initiating structural transitions from the OC to IF state. Koldsø et al. has shown that solvation of the intracellular vestibule allows Asp437 to rotate Na2 towards the intracellular pathway and facilitates transitions to the IF conformation. ${ }^{78}$ Similarly, we also observed that the rotameric shift in Asp437 initiates the dissociation of $\mathrm{Na}^{+}$from the $\mathrm{Na} 2$ site (Figure S26A, S26B). The $\mathrm{Na}^{+}$ion then interacts with Ser91 and is further stabilized by Phe347 and Phe440 via cation- $\pi$ interactions (Figure S26C). Finally, the $\mathrm{Na}^{+}$ion engages with Asp87 and diffuses into the intracellular space (Figure S26D). Koldsø et al. also predicted that Glu444 interacts with Na2 in the exit pathway,. ${ }^{78}$ yet in our simulations, Glu444 forms an ionic interaction with Arg462 and does not interact with Na2. At this juncture, the intracellular gating residues (Arg79 and Asp452) still hold their hydrogen bond interactions, and $\mathrm{Na}^{+}$can access the intracellular pathway 

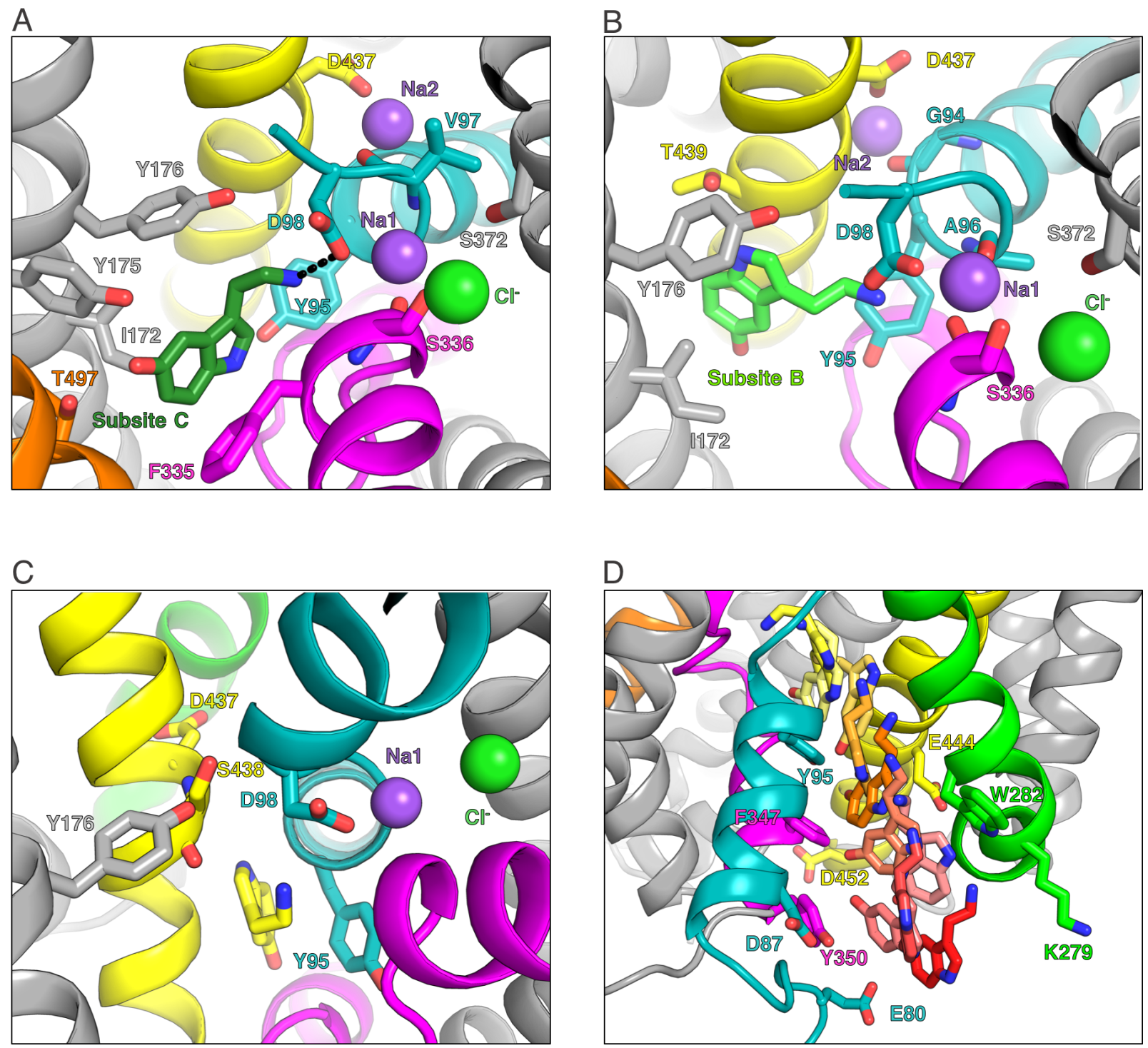

Figure 6: MD snapshots of $5 \mathrm{HT}$ in the orthosteric binding site and intracellular release. Transmembrane helices 1, 5, 6, 8, and 10, are colored in teal, green, magenta, yellow, and orange, respectively. Substrate color relates to progression of 5HT-import as shown in Figure 5A. (A,B) While 5HT is in subsite $\mathrm{C}$ and B, the amine moiety of $5 \mathrm{HT}$ at the orthosteric site interacts with Asp98, disrupting the hydrogen bonding interaction between Tyr176-Asp98. (C) The rotameric flip of the phenol ring of Tyr95 initiates the opening of the intracellular vestibule and allows for permeation of $5 \mathrm{HT}$ towards the intracellular exit pathway. (D) $5 \mathrm{HT}$ translocation through the exit pathway between TM1a and TM5. 
without the breakage of ionic contacts. However, in $\mathrm{Na}^{+}$-SERT simulations, the $\mathrm{Na} 2$ ion has a tendency to rebind to the Na2 site after release (Figure S27). Such intracellular binding events were not observed in 5HT-SERT simulations. The coupling of 5HT import to the cytoplasmic release of $\mathrm{Na}^{+}$from the Na2 site explains the 1:1 sodium to neurotransmitter stoichiometry of the transport cycle.

The rotameric shift of Tyr95 results in permeation of 5HT to the exit pathway (Figure 6C). The flipping of the indole ring of 5HT displaces the ionic interactions with Asp98 resulting in the aromatic ring of 5HT to be trapped between Tyr95 and Val343. The 'downward' movement of 5HT disrupts contacts between TM1a and TM5, specifically the hydrogen bonding interactions between Asp87 and Trp282 (Figure S9). The distance between Asp87 and Trp282 increases up to $16 \AA$, thus drives the dissociation of $5 \mathrm{HT}$ to the cytoplasmic half of the transporter. Additionally, the intracellular salt bridge network between Glu80-Lys279 and Arg79-Asp452 weakens as the cytoplasmic base of TM5 unwinds, further resulting in the opening of the intracellular pathway. 5HT shifts to the intracellular vestibule and occupies the Na2 site (Figure 6C). The amine group forms strong polar contacts with residues in the Na2 site, and the indole ring is lodged between Tyr95, Phe347, and Phe440. 5HT further diffuses down, however the amine group of 5HT still forms interactions with Tyr95, and the indole-NH forms additional interactions with Ser91. Finally, 5HT leaves the transporter through a widened intracellular pathway surrounded by TM1a and TM5. Our results show that the rotation of Tyr95 propagates the opening of TM1a and mediates substrate transport to the cytoplasmic half. The equivalent residue in $h \mathrm{DAT}$, Phe76, has been shown to undergo a similar rotameric transition to allow for substrate release. ${ }^{40}$ We also observed that the cytoplasmic base of TM5 unwinds and shifts outward by $\sim 8-10 \AA$ to facilitate cytoplasmic opening of the exit pathway (Figure 6D), and it is well known that these regions play a crucial role in regulating SERT activity ${ }^{64}$. 


\section{Simulations identify key residues involved in substrate transport.}

SERT has been extensively studied by targeted mutagenesis, especially within the orthosteric binding site, confirming key interacting residues with substrate and drugs. ${ }^{84,89}$ To provide basic evidence supporting aspects of the simulations, we instead focused mutagenesis to residues predicted to make early interactions with substrate (Figure 7A). Changes in transport activity were assessed based on cellular uptake of a fluorescent 5HT analogue, APP+, which has been shown to have similar transport properties to $5 \mathrm{HT}^{90}$ Furthermore, we extracted individual states from the 5HT-SERT simulation data and performed molecular docking experiments to predict the binding mode of $\mathrm{APP}+$, and observed that $\mathrm{APP}+$ binds in a similar fashion and shares similar residue interactions as the native substrate, 5HT (Figure S28).

We examined by immunoblot that the decreased transport activity was not a result due to poor SERT expression, with the exception of Ile108Ala (Figure 7B). Alanine substitutions of Tyr107 and Gln111, which are predicted to participate in the early recognition of 5HT as it first diffuses into the extracellular vestibule and substitution of Asp328 in the allosteric site reduce substrate transport (Figure 7B). Both mutations are deleterious for transport activity as they mediate crucial interactions with the substrate in the initial recognition as well as in the allosteric site (Figure 7B and 7C). Leu502 is packed beneath aromatic residues in the allosteric and early recognition sites, and disruption of local structure by its substitution to alanine renders SERT inactive, although we cannot exclude broader effects on the protein fold (Figure 7D). We further tested alanine substitution of Val343, which is packed against Tyr95 at the base of the orthosteric site. Once Tyr95 undergoes a rotameric shift during the OC to IF transition, a void is created between Val343 and Tyr95 that is temporarily occupied by the substrate as it moves into the intracellular vestibule (Figure 7E). SERT Val343Ala has partly reduced activity, which is notable considering the close chemical similarity between alanine and valine side chains. Additionally, we determined the $\mathrm{K}_{\mathrm{M}}-\mathrm{V}_{\mathrm{Max}}$ values for a mutant in each interaction site which show a reduction in transport activity (Table S2). Finally, Glu136 and Glu508 that coordinate $\mathrm{Na}^{+}$in the Na3 site were 
A
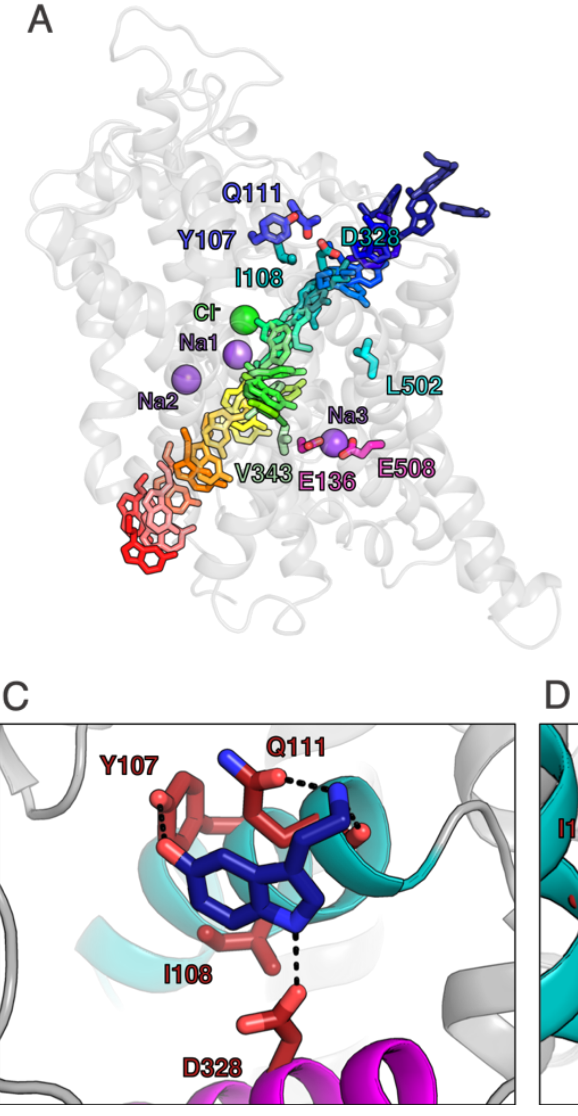

B
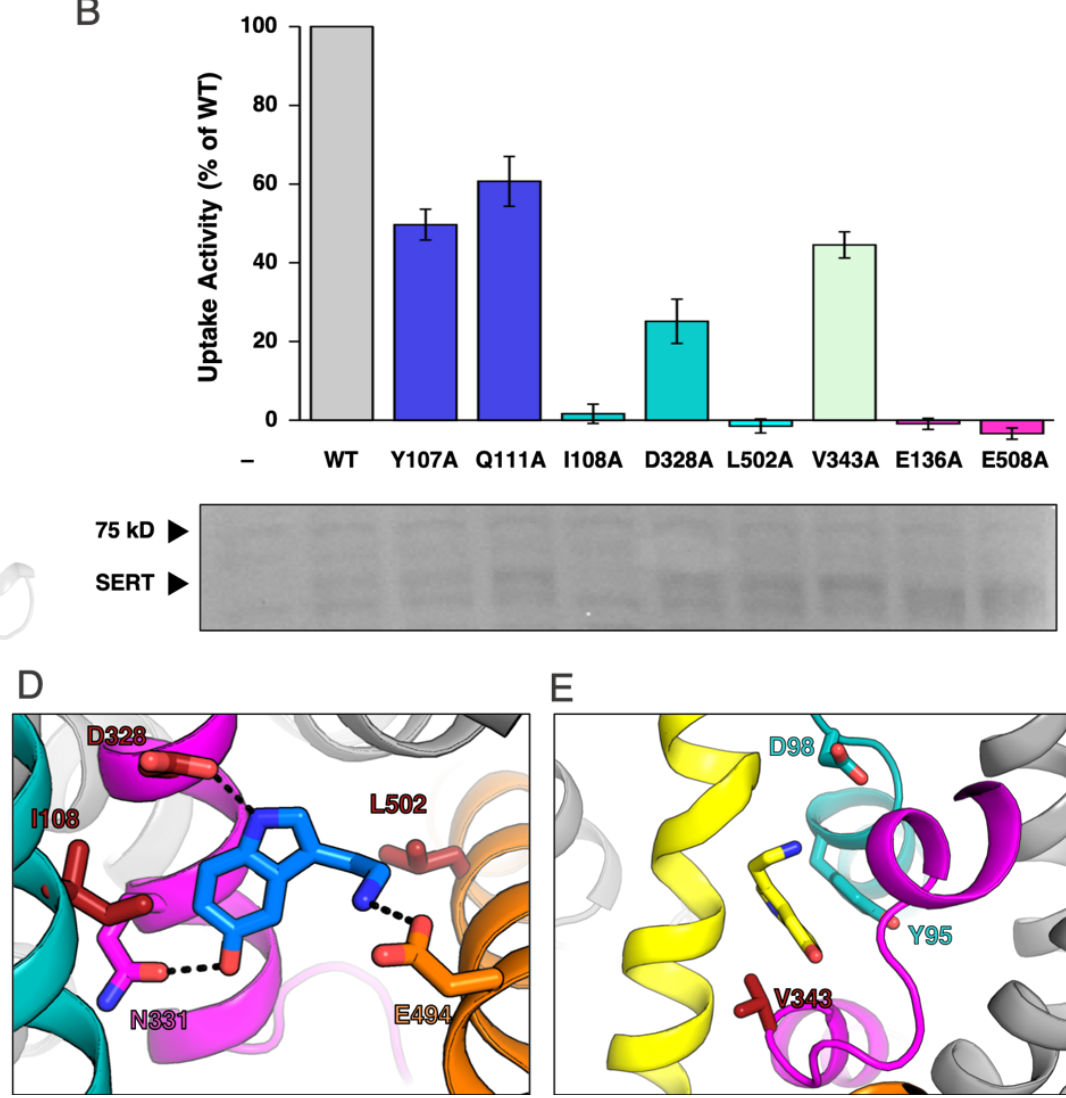

E

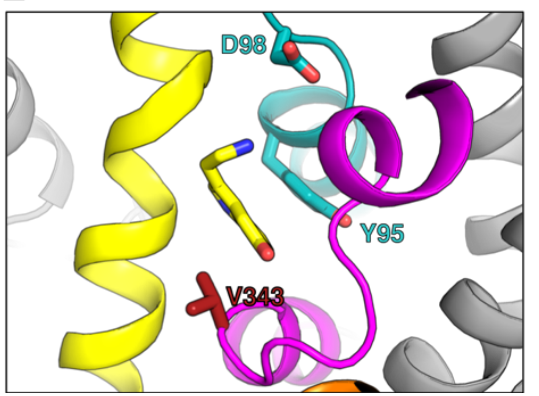

Figure 7: Alanine substitutions of residues involved in the simulated transport process cause decreased substrate uptake. (A) Overlaid MD snapshots showing the permeation of 5HT from its diffusion into the extracellular vestibule (dark blue), to binding at the orthosteric site (green), to cytosolic release (red). Mutated residues are similarly colored based on when in the import pathway they interact with $5 \mathrm{HT}$. Glutamates coordinating $\mathrm{Na}^{+}$in the $\mathrm{Na} 3$ site are magenta. (B) SERT mutants were expressed in human Expi293F cells and uptake of fluorescent substrate $\mathrm{APP}+(0.5 \mu \mathrm{M})$ was compared to wild type (WT) protein. Data are mean $\pm \mathrm{SD}, \mathrm{n}=3$. Aligned below is an anti-SERT immunoblot of total cell lysate. (C-E) MD snapshots of 5HT at the (C) extracellular vestibule, (D) allosteric site, and (E) released from the orthosteric site. Residues that were substituted with alanine colored as red sticks. Transmembrane helices 1, 6, 8, and 10, are colored in teal, magenta, yellow, and orange, respectively.

mutated to alanine to cause a complete loss of activity, demonstrating the importance of this region for the transport process. 


\section{Conclusions}

In this study, we present an atomistic view of the substrate import process in SERT as well as characterizing the thermodynamics of key states involved in substrate transport. By implementing an MSM-based adaptive sampling protocol to sample the conformational landscape, we investigated global transitions from OF to IF for both 5HT-free and 5HTtransporting SERT. MSM-weighted conformational free energy landscapes show the OF and OC states are relatively stable, and transitions to and from OF and OC states are relatively low energy. Transitions from OC to IF are substantially higher, with energy barriers of $\sim 2 \mathrm{kcal} / \mathrm{mol}$ in $\mathrm{Na}^{+}-\mathrm{SERT}$; however, the presence of $5 \mathrm{HT}$ not only lowers the free energy barrier of OF to OC transitions, but further stabilizes SERT in the IF state as compared to $\mathrm{Na}^{+}$-SERT. The dependence of the IF state on the presence of substrate has experimentally been observed for the bacterial LeuT transporter, where addition of extracellular substrate promotes dynamics at the intracellular gate. ${ }^{62}$

The simulated structures show similar helix orientations at the extracellular and intracellular gates with respect to experimental SERT structures. ${ }^{23,44,46}$ The comparison of $\mathrm{Na}^{+}$and 5HT-SERT data reveal that the structural transitions from OF to OC involve minimal helix movements in TM1b, TM6a, and TM10, while OC to IF transitions show higher fluctuations of intracellular helix tips of TM1a, TM5, and TM7 to facilitate opening of the intracellular vestibule (Figure S4). Biochemical experiments in conjunction with computational modeling have shown that cholesterol binding to a conserved site comprised of TM1a, TM5, and TM7 inhibits these helix movements and may act as a regulatory mechanism for SERT function. ${ }^{46,91,92}$ Deviations were observed in the opening of the intracellular vestibule of the IF state between the cryo-EM and predicted MD structure, specifically the outward motion of TM1a. This might reflect the loss of lateral pressure following detergent-extraction; the membrane is anticipated to constrain the extent to which TM1a can move away from the helical bundle. Nevertheless, our results show that partial opening of the intracellular pathway is sufficient for substrate transport. Experimental studies of LeuT also indicate that a 
partially-open IF conformation is suitable for substrate transport. ${ }^{28}$

Conducting simulations of both serotonin-free and serotonin-present conditions allows us to examine the finer details of the substrate-induced conformational transitions and the cooperative nature between the transporter and the substrate. Motions of TM2 were highly correlated with TM1b in $\mathrm{Na}^{+}-\mathrm{SERT}$, whereas the binding of $5 \mathrm{HT}$ decreases the cooperativity as TM1b rotates and closes the extracellular cavity to open the exit pathway (Figure S29). The conformation dynamics examined through HDX studies reveal that TM2 undergoes less fluctuations when bound to $\mathrm{Na}^{+}$, in contrast to when the transporter is substrate bound. ${ }^{54}$ Similar trends were observed between TM6a and TM2, more specifically, the presence of 5HT destabilizes this region and favors the conformational change required for substrate transport (Figure S29). Gating residues Arg104-Glu493 remain open until the ions and 5HT bind to the primary binding site, and later close the extracellular cavity as the exit pathway opens to allow the release of substrate molecules (Figure S5). At the binding site, Tyr176 has the tendency to form a polar interaction with Asp98 in the OF and OC states. Interactions by the amine moiety of the substrate sever the Tyr176-Asp98 interaction, which is further disrupted when the opening of the intracellular cavity induces a rotameric shift in Tyr176 away from the Asp98 (Figure S19). Most significantly, the closure of the extracellular allosteric cavity is coupled to opening of the intracellular helix TM1a when the substrate is bound (Figure S29C). Experimental studies show that the substrate binding increases dynamics between EL4 and TM12. ${ }^{24,54-56}$ In simulations, EL4 inserts deep inside the extracellular cavity, which is further facilitated by the movement of TM12 (Figure S30). Furthermore, HDX studies on DAT support the coupled motions between TM7 and TM12 observed in simulations when the substrate is bound, compared to just $\mathrm{Na}^{+}$binding (Figure S29).

Our simulations reveal an ordered sequence of binding and transport events that agree with the 1:1 substrate: $\mathrm{Na}^{+}$stoichiometry as previously characterized for the NSS family. In 5HT-SERT simulations when $\mathrm{Na} 1$ and $\mathrm{Na} 2$ sites are occupied, both $\mathrm{Cl}^{-}$ions and 5HT molecules were present in the solution, but even after extensive sampling, we only observed 
5HT binding to the transporter. Similarly, in the $\mathrm{Na}^{+}-\mathrm{SERT}$ simulations, $\mathrm{Na}^{+}$and $\mathrm{Cl}^{-}$ions were present in the solution, yet we observed rapid diffusion and binding of $\mathrm{Na}^{+}$ions to their binding sites instead of $\mathrm{Cl}^{-}$ion binding. We further determined that the free energy barrier for $\mathrm{Cl}^{-}$to bind without the substrate in the orthosteric cavity is significantly greater than in 5HTSERT simulations (Figure S25). Additionally, the calculated electrostatic potential map of the OF SERT cryo-EM structure reveals that the permeation pathway is negatively charged and supports our finding that the binding of $\mathrm{Cl}^{-}$prior to substrate binding will be associated with a large free energy barrier (Figure S15). We identified a third sodium ion binding site buried deep in the binding pocket that is conserved among various NSS transporters and critical for substrate transport. Similar to other NSS members, the release of $\mathrm{Na}^{+}$from the Na2 site decouples the interactions between TM1a, TM5, and TM8, increasing the solvation of the intracellular permeation pathway and allowing TM1a to open for substrate release. As unbiased molecular dynamics simulations can only sample events with low free energy barriers, we have the highest likelihood of observing the most probable binding sequence of ions and substrate. However, we do not disregard that different sequences of ion and substrate binding events with higher free energy barriers associated with them are possible and may alter the kinetics of the SERT transport cycle. Differences in stoichiometry may arise if the ion unbinding timescales for $\mathrm{Na} 1, \mathrm{Na} 3$, and $\mathrm{Cl}^{-}$are much longer than the simulated timescales obtained in this study. We investigated the mechanism of 5HT recognition, binding, and translocation in SERT and through mutagenesis provided support for certain residues having potential roles in substrate binding and/or conformational transitions, though we do not exclude that they may have other roles in folding of the transport cycle.

In some simulations, we observed 5HT to simultaneously bind both the orthosteric and allosteric site. Substrate bound at the allosteric site hinders the fluctuations of several residues at the extracellular side, hence, allosterically communicates to the primary binding site of the transporter by limiting conformational transitions from the 5HT-bound OF state. The distance distribution plot reveals that the side chain conformation of Arg104 is restricted 
when $5 \mathrm{HT}$ binds to both orthosteric and allosteric sites, whereas when $5 \mathrm{HT}$ is solely occupied the orthosteric site, Arg104 may adopt multiple conformations (Figure S31). The binding of the substrate at both sites sterically restricts opening of the exit permeation and substrate release.

This study has explored the conformational dynamics and substrate import of a monomeric unit of SERT; however, fluorescent microscopy has shown SERT to form functional oligomers in the membrane. ${ }^{93,94}$ The LeuT crystal structure has been resolved as a dimeric unit, and while recent modeling studies have explored possible dimeric SERT units ${ }^{95}$ the exact interface of SERT oligomerization and the effects of coupled dynamics remains unclear. Further investigation is required into understanding how in vivo regulation affects the kinetics and conformational landscape of SERT.

\section{Methods}

\section{Molecular dynamics (MD) simulations.}

The OF SERT crystal structure (PDB: 5I73) was used as the starting model for MD simulation. ${ }^{44}$ Thermostable mutations in the crystal structure Ala110, Ala291, and Ser439 were reverted to wild type Tyr110, Ile291, and Thr439, respectively. The protein was embedded in a phosphatidylcholine (POPC) bilayer with CHARMM-GUI ${ }^{96}$ and solvated with TIP3P water molecules. ${ }^{97} 150 \mathrm{mM} \mathrm{NaCl}$ was added to neutralize the system and mimic physiological conditions. Terminal chains were capped with acetyl and methyl amide groups. Overall, the final Na+-SERT system consisted of $\sim 70,000$ atoms in a period box volume of $77 \mathrm{X} 77 \mathrm{X}$ $113 \AA^{3}$. The MD system was built using the tleap module of AMBER14. ${ }^{98}$ The MD system was minimized for 20,000 steps using the conjugate gradient method, heated from 0 to 300 $\mathrm{K}$ at NVT, and equilibrated for $40 \mathrm{~ns}$ under NPT conditions. $\mathrm{A} \mathrm{Na}{ }^{+}$bound in the Na1 and $\mathrm{Na} 2$ site OF SERT structure, obtained from $\mathrm{Na}^{+}$-SERT simulations, was used as the starting model to capture the mechanism of $5 \mathrm{HT}$ import. $100 \mathrm{mM}$ serotonin (equivalent to 12 
5HT molecules was randomly added to the simulation box and equilibrated under the same conditions described previously. We note that the simulated 5HT concentration is unphysiological and was implemented to enhance the probability of substrate binding events. During the alternate-access cycle, the transporter only interacts with at most two 5HT molecules. Therefore, the free energy and kinetics of the conformational change processes are independent of the additional 5HT molecules in solution. The ionic charge of 5HT is neutralized with $\mathrm{Cl}^{-}$ions. While the ionic concentration could have some effects on the protein conformation such as altering the structure of the extracellular loops which directly interact with the additional 5HT molecules, we do not observe any shifts in the structure of these loops between the $\mathrm{Na}^{+}$-SERT and 5HT-SERT simulations. All simulations were implemented using Amber14 package ${ }^{98-101}$ employing Amber ff14SB ${ }^{102}$ force field combined with GAFF force field at constant NPT conditions (300K, $1 \mathrm{~atm})$ and periodic boundary conditions. Temperature and pressure were maintained with Berendsen thermostat and barostat, respectively. ${ }^{103}$ Electrostatic interactions were treated with the Partial Mesh Ewald method, ${ }^{104}$ and hydrogen bonds were constrained using SHAKE algorithm. ${ }^{105}$ Nonbonded distance cutoff was set at $10 \AA$, and an integration timestep of $2 \mathrm{fs}$ was used for all simulations. Snapshots were saved every 100 ps during production simulations.

\section{Adaptive sampling.}

Obtaining sufficient sampling is a reoccurring challenge in simulating complex biological processes. To overcome this issue, we adopted a Markov state model (MSM)-based adaptive sampling methodology to efficiently explore the conformational landscape. ${ }^{57,106,107}$ In each round of adaptive sampling, multiple short MD simulations are conducted in parallel. The simulation data is clustered using the K-means algorithm ${ }^{108}$ based on a designated metric and starting structures are chosen from the least populated states to seed the subsequent rounds of simulation. The sampling bias introduced from least-count selection is eliminated during the construction of the MSM by estimating the reverse transition probability matrix 
for transition between all conformational states. In the limit of long timescales, the sampling errors are expected to be small. We show that there is little difference between the population of states derived from the raw data and the MSM-derived equilibrium population on the conformational landscape (Figure S32). Extracellular and intracellular gating residues were used as adaptive sampling metrics for the conformational sampling of the states and zposition of substrates to capture the import process. A total of $\sim 130 \mu \mathrm{s}$ of $\mathrm{Na}^{+}$- and $\sim 210$ $\mu$ s $5 \mathrm{HT}$ transport simulation data was obtained. Multiple substrate binding and transport events were captured as a direct advantage of the MSM-based adaptive sampling approach (Figure S33). The entire MD dataset was used for MSM construction and analysis.

\section{MSM construction.}

The CPPTRAJ and pytraj ${ }^{109}$ modules in AmberTools and MDTraj Python library ${ }^{110}$ were used for post processing the trajectory data. Markov state models (MSMs) were constructed using pyEMMA 2.5.6 Python package. ${ }^{111}$ MSMs were constructed for both $\mathrm{Na}^{+}-$and $5 \mathrm{HT}$ transport datasets. 15 residue-residue pair distances surrounding the permeation pathway for clustering (Figure S34). Additionally, the z-components of the $2 \mathrm{Na}^{+}$ions were incorporated to the 15 residue-residue pair distances as featurization metrics for $\mathrm{Na}^{+}$-SERT simulations. The z-components of $5 \mathrm{HT}, \mathrm{Cl}^{-}$, and the symported $\mathrm{Na}^{+}$ion were added along with the 15 distances for the 5HT transport process. Time-structure independent component analysis (tICA) was performed on the feature matrix to reduce the dimension space by obtaining the slowest-relaxing degrees of freedom as a linear combination of the features. ${ }^{112}$ The optimum number of clusters and time-independent components (tICs) were obtained from the set that yielded the greatest sum of the eigenvalues of the transition matrix, also known as the VAMP1 score. 700 clusters and 8 tICs were used to construct the MSM for $\mathrm{Na}^{+}$SERT simulations. 700 clusters and 10 tICs were used for 5HT-SERT MSM. (Figure S35A). The lag time of $8 \mathrm{~ns}$ was determined for MSM construction from implied timescale plots (Figure S35B). The Chapman-Kolmogorov test, which validates the Markovian behavior of 
the MSM, ${ }^{113}$ was performed on 5 macrostates implemented in pyEMMA (Figure S36, S37).

\section{Trajectory analysis.}

MSM-weighted simulation data were plotted on the coordinates of the gating distances, specifically the distances between the closest heavy atom between Arg104 and Glu493 of the extracellular gate, and the closest heavy atom between groups of residues Gly77-Ser91 of the N-terminus and TM1a and Lys297-Trp282 of TM5 for the intracellular gate (Figure S1). MSM states were further clustered into macrostates and visualized with Visual Molecular Dynamics (VMD) ${ }^{114}$ and PyMOL (Schrödinger, LLC). The predicted fraction deuteration was calculated according to Adhikary et al. ${ }^{24}$ on $1000 \mathrm{OF}$ and IF structures randomly extracted from the $\mathrm{Na}^{+}-$SERT and 5HT-SERT MSM. The 5HT-residue interactions were obtained from python scripts implemented from the GetContacts package (https://getcontacts.github.io/). To calculate the RMSF between transitions, 1000 structures of each OF, OC, and IF states were randomly extracted from the MSM and measured with respect to the cryo-EM structure of the prior conformational state (i.e. OF-OC: 1000 OC structures with respect to OF cryoEM structure). In-house scripts and matplotlib Python library were used to generate plots. Channel pore radius was calculated using the HOLE program. ${ }^{115}$ pKa values were calculated using DelPhiPKa program as it also considers the effects of solute molecules while preforming the calculation in the static structure. ${ }^{116}$ Cross correlation analysis of $\mathrm{Na}^{+}$-SERT and 5HT-SERT was performed to identify the allosteric effects of substrate on conformational changes between various states and the substrate transport. The cross-correlation value of 0 represents motions that are not correlated, while a value of 1 shows that the motions are highly coupled.

\section{Transition path theory.}

The top flux pathways for conformational changes and 5HT import were determined using transition path theory (TPT) analysis. Given a transition matrix obtained from the Markov 
state model, TPT examines the transition probabilities and estimates transitions pathways connecting the source (defined as state $A$ ) and sink (defined as state $B$ ) states and the fluxes associated with the pathway. ${ }^{117}$ The flux between state $i$ to $j$ or $f_{i j}$ is defined as

$$
f_{i j}=\pi_{i} q_{i}^{-} T_{i j} q_{j}^{+}
$$

where $\pi_{i}$ is the stationary probability of state $i$ as obtained from the eigenvalue decomposition of the transition matrix, $T_{i j}$ is the probability of transitioning from state $i$ to state $j, q_{i}^{-}$ is the backwards committer probability of transitioning to the source state rather than the sink state from state $i$, and $q_{j}^{+}$is the forwards committer probability of transitioning to the sink state rather than the source state from state $j .{ }^{118}$ The forward committer probability for a state $i$, or $q_{i}^{+}$is computed as the sum of all the transitions from state $i$ the sink state $B$ and the sum of all the transitions from state $i$ to sink state $B$ via an intermediate state $I,{ }^{118}$ or

$$
q_{i}^{+}=\sum_{k \in B} T_{i k}+\sum_{k \in I} T_{i k} q_{k}^{+}
$$

The backwards probability committer probability is calculated as

$$
q_{i}^{-}=1-q_{i}^{+}
$$

TPT analysis was conducted using the pyEMMA 2.5.6 Python package. ${ }^{111}$

\section{Umbrella sampling of $\mathrm{Cl}^{-}$binding in $\mathrm{Na}^{+}$-SERT}

Umbrella sampling MD simulations were used to calculate the binding free energy of $\mathrm{Cl}$ to the $\mathrm{Cl}^{-}$site without the presence of the substrate. The $\mathrm{Cl}^{-}$binding reference pathway was obtained from 5HT-SERT simulations. Using the distance of $\mathrm{Cl}^{-}$to the binding site as the collective variable, 73 equally spaced umbrella sampling windows from 0 to $18 \AA$ 
at $0.25 \AA$ intervals. At each window, the $\mathrm{Cl}^{-}$ion was restrained by a harmonic potential with spring constant $10 \mathrm{kcal} / \mathrm{mol}-\AA^{2}$. The umbrella sampling simulations were implemented using the Amber18 package. ${ }^{119}$ Each window was equilibrated for $10 \mathrm{~ns}$ and followed by 25 ns production. The free energy was constructed using the weighted histogram analysis (WHAM) software ${ }^{120}$ and averaged over 5 independent runs.

\section{Plasmid construction.}

Human SERT was PCR amplified from pcDNA3- $h$ SERT (Addgene 15483$)^{121}$ to introduce a consensus Kozak sequence and ligated into the Kpn1-Xho1 sites of pCEP4 (Invitrogen). Alanine substitutions were introduced by overlap extension PCR, and all plasmid sequences were verified.

\section{Transport Assay.}

Expi293F cells (ThermoFisher) were cultured in Expi293F expression medium (ThermoFisher) at $37^{\circ} \mathrm{C}, 8 \% \mathrm{CO}_{2}, 125 \mathrm{rpm}$, and transfected with $500 \mathrm{ng}$ plasmid per ml at a density of $2 \times 10^{6}$ cells/ml using Expifectamine (ThermoFisher). 24-28 hr post-transfection, cells were washed with PBS-BSA (Dulbecco's phosphate-buffered saline supplemented with $0.2 \%$ bovine serum albumin), incubated with fluorescent substrate analogue APP+ (Aobious) in PBS-BSA for 2 min at room temperature, washed twice with ice cold PBS-BSA, and analyzed on a BD LSRII cytometer. The main cell population was gated by forward and side scatter properties to exclude debris and doublets, and 10,000 gated events were collected. Cell fluorescence was measured with a 488nm laser, 505 LP dichroic mirror, and 530/30 bandpass filter. Fluorescence corresponding to non-specific uptake by vector-transfected cells was subtracted, and activity of mutants was assessed relative to the wild type construct. 


\section{Immunoblot.}

Cells were collected $24 \mathrm{hr}$ post-transfection, solubilized in SDS loading dye, and proteins were separated by SDS-polyacrylamide gel electrophoresis. After transferring proteins to polyvinylidene fluoride membrane (Bio-rad) at $4^{\circ} \mathrm{C}$, the membrane was blocked for $1 \mathrm{hr}$ at room temperature with tris-buffered saline containing $0.1 \%$ Tween 20 (TBST) and 3\% skim milk powder. The membrane was incubated with 1:1,000 anti-SERT rabbit polyclonal (Abcam Cat. No. ab102048) in TBST containing 1\% milk overnight at $4^{\circ} \mathrm{C}$, washed 3 times with TBST for $5 \mathrm{~min}$, and incubated with 1:10,000 HRP conjugated goat anti-rabbit IgG (Jackson ImmunoResearch Laboratories) in TBST containing 1\% milk for $1 \mathrm{hr}$ at room temperature. The membrane was washed three times with TBST for $5 \mathrm{~min}$, and developed using clarity western ECL substrate (Bio-rad). The signal was visualized using Gel Doc (Bio-rad).

\section{Molecular Docking.}

Docking was performed using AutoDock 4.2. ${ }^{122}$ The PDBQT format files for the SERT structures and APP+ was obtained using AutoDock python utility modules. The grid center was chosen by selecting the residues within $6 \AA$ of the ligand and grid maps were generated using AutoGrid. The ligands were docked into the active site using AutoDock. The default Lamarkian Genetic algorithm was used for performing energy evaluation of the ligand conformations. ${ }^{123}$ The docked poses were visualized in PyMOL (Schrödinger, LLC).

\section{Author Information}

Corresponding Author

Email: diwakar@illinois.edu, procko@illinois.edu 


\section{Funding sources}

This work was funded by NSF MCB 18-45606 to DS. EP is supported by R21 MH113155 from NIMH.

\section{Notes}

The authors declare no competing financial interest.

\section{Acknowledgement}

The authors thank the Blue Waters sustained-petascale computing project, which is supported by the National Science Foundation (awards OCI-0725070 and ACI-1238993) and the state of Illinois. Blue Waters is a joint effort of the University of Illinois at UrbanaChampaign and its National Center for Supercomputing Applications.

\section{Abbreviations}

SERT, serotonin transporter; 5HT, 5-hydroxytryptamine; DAT, dopamine transporter; NET, norepinephrine transporter; NSS, neurotransmitter:sodium symporter; TM, transmembrane; LeuT, leucine transporter; OF, outward-facing; OC, occluded; IF, inward-facing; EPR, electron paramagentic resonance; smFRET, single-molecule fluorescence resonance energy transfer; dDAT, Drosophila dopamine transporter; hSERT, human serotonin transporter; EL, extracellular loop; HDX, hydrogen-deuterium exchange; MSM, Markov state model; APP+, 4-(4-dimethylamino)phenyl-1-methylpyridinium.

\section{References}

(1) Berger, M.; Gray, J. A.; Roth, B. L. The expanded biology of serotonin. Annu. Rev. Med. 2009, 60, 355-366. 
(2) Murphy, D. L.; Lerner, A.; Rudnick, G.; Lesch, K.-P. Serotonin transporter: gene, genetic disorders, and pharmacogenetics. Mol. Interv. 2004, 4, 109.

(3) Ni, W.; Watts, S. W. 5-Hydroxytryptamine in the Cardiovascular System: Focus on the Serotonin Transporter (SERT). Clin. Exp. Pharmacol. Physiol. 2006, 33, 575-583.

(4) Gether, U.; Andersen, P. H.; Larsson, O. M.; Schousboe, A. Neurotransmitter transporters: molecular function of important drug targets. Trends Pharmacol. Sci. 2006, 27, 375-383.

(5) Rudnick, G.; Wall, S. C. The molecular mechanism of "ecstasy" [3,4-methylenedioxymethamphetamine (MDMA)]: serotonin transporters are targets for MDMA-induced serotonin release. Proc. Natl. Acad. Sci. U.S.A. 1992, 89, 1817-1821.

(6) Nelson, P. J.; Rudnick, G. The role of chloride ion in platelet serotonin transport. J. Biol. Chem. 1982, 25\%, 6151-6155.

(7) Rudnick, G.; Nelson, P. J. Platelet 5-hydroxytryptamine transport, an electroneutral mechanism coupled to potassium. Biochemistry 1978, 17, 4739-4742.

(8) Rudnick, G. Active transport of 5-hydroxytryptamine by plasma membrane vesicles isolated from human blood platelets. J. Biol. Chem. 1977, 252, 2170-2174.

(9) Hasenhuetl, P. S.; Freissmuth, M.; Sandtner, W. Electrogenic binding of intracellular cations defines a kinetic decision point in the transport cycle of the human serotonin transporter. J. Biol. Chem. 2016, 291, 25864-25876.

(10) Rudnick, G.; Sandtner, W. Serotonin transport in the 21st century. J. Gen. Physiol. 2019, 151, 1248-1264.

(11) Lin, F.; Lester, H. A.; Mager, S. Single-channel currents produced by the serotonin transporter and analysis of a mutation affecting ion permeation. Biophys. J 1996, 71, 3126-3135. 
(12) Mager, S.; Min, C.; Henry, D. J.; Chavkintt, C.; Hoffman, B. J.; Davidson, N.; Lester, H. A. Conducting states of a mammalian serotonin transporter. Neuron 1994, 12, 845-859.

(13) Ruchala, I.; Cabra, V.; Solis, E.; Glennon, R. A.; De Felice, L. J.; Eltit, J. M. Electrical coupling between the human serotonin transporter and voltage-gated Ca2+ channels. Cell Calcium 2014, 56, 25-33.

(14) Quick, M. W. Regulating the Conducting States of a Mammalian Serotonin Transporter. Neuron 2003, 40, 537-549.

(15) Keyes, S. R.; Rudnick, G. Coupling of transmembrane proton gradients to platelet serotonin transport. J. Biol. Chem. 1982, 257, 1172-1176.

(16) Forrest, L. R. Structural Symmetry in Membrane Proteins. Annu. Rev. Biophys. 2015, $44,311-337$.

(17) Yamashita, A.; Singh, S. K.; Kawate, T.; Jin, Y.; Gouaux, E. Crystal structure of a bacterial homologue of $\mathrm{Na}+/ \mathrm{Cl}$ - -dependent neurotransmitter transporters. Nature 2005, 437, 215.

(18) Chen, J.-G.; Rudnick, G. Permeation and gating residues in serotonin transporter. Proc. Natl. Acad. Sci. U.S.A. 2000, 97, 1044-1049.

(19) Quick, M.; Javitch, J. A. Monitoring the function of membrane transport proteins in detergent-solubilized form. Proc. Natl. Acad. Sci. U.S.A. 2007, 104, 3603-3608.

(20) Mitchell, P. A General Theory of Membrane Transport From Studies of Bacteria. Nature 1957, 180, 134-136.

(21) Forrest, L. R.; Zhang, Y.-W.; Jacobs, M. T.; Gesmonde, J.; Xie, L.; Honig, B. H.; Rudnick, G. Mechanism for alternating access in neurotransmitter transporters. Proc. Natl. Acad. Sci. U.S.A. 2008, 105, 10338-10343. 
(22) Jardetzky, O. Simple Allosteric Model for Membrane Pumps. Nature 1966, 211, 969.

(23) Krishnamurthy, H.; Gouaux, E. X-ray structures of LeuT in substrate-free outwardopen and apo inward-open states. Nature 2012, 481, 469-474.

(24) Adhikary, S.; Deredge, D. J.; Nagarajan, A.; Forrest, L. R.; Wintrode, P. L.; Singh, S. K. Conformational dynamics of a neurotransmitter:sodium symporter in a lipid bilayer. Proc. Natl. Acad. Sci. U.S.A. 2017, 114, E1786-E1795.

(25) Gur, M.; Zomot, E.; Cheng, M. H.; Bahar, I. Energy landscape of LeuT from molecular simulations. J. Chem. Phys. 2015, 143, 243134.

(26) Shi, L.; Quick, M.; Zhao, Y.; Weinstein, H.; Javitch, J. A. The Mechanism of a Neurotransmitter:Sodium SymporterInward Release of $\mathrm{Na}+$ and Substrate IsTriggered by Substrate in a Second Binding Site. Mol. Cell 2008, 30, 667-677.

(27) Singh, S. K.; Pal, A. In Methods in Enzymol; Shukla, A. K., Ed.; Membrane ProteinsEngineering, Purification and Crystallization; Academic Press, 2015; Vol. 557; pp $167-198$.

(28) Terry, D. S.; Kolster, R. A.; Quick, M.; LeVine, M. V.; Khelashvili, G.; Zhou, Z.; Weinstein, H.; Javitch, J. A.; Blanchard, S. C. A partially-open inward-facing intermediate conformation of LeuT is associated with $\mathrm{Na}+$ release and substrate transport. Nat. Commun. 2018, 9, 230.

(29) Claxton, D. P.; Quick, M.; Shi, L.; de Carvalho, F. D.; Weinstein, H.; Javitch, J. A.; Mchaourab, H. S. Ion/substrate-dependent conformational dynamics of a bacterial homolog of neurotransmitter:sodium symporters. Nat. Struct. Mol. Biol. 2010, 17, $822-829$.

(30) Kazmier, K.; Sharma, S.; Islam, S. M.; Roux, B.; Mchaourab, H. S. Conformational 
cycle and ion-coupling mechanism of the $\mathrm{Na}+$ /hydantoin transporter Mhp1. Proc. Natl. Acad. Sci. U.S.A. 2014, 111, 14752-14757.

(31) Kazmier, K.; Sharma, S.; Quick, M.; Islam, S. M.; Roux, B.; Weinstein, H.; Javitch, J. A.; Mchaourab, H. S. Conformational dynamics of ligand-dependent alternating access in LeuT. Nat. Struct. Mol. Biol 2014, 21, 472-479.

(32) Focke, P. J.; Wang, X.; Larsson, H. P. Neurotransmitter Transporters: Structure Meets Function. Structure 2013, 21, 694-705.

(33) Shaikh, S. A.; Tajkhorshid, E. Modeling and dynamics of the inward-facing state of a $\mathrm{Na}+/ \mathrm{Cl}-$ dependent neurotransmitter transporter homologue. PLoS Comput. Biol. 2010, 6, e1000905.

(34) Zhao, Y.; Terry, D.; Shi, L.; Weinstein, H.; Blanchard, S. C.; Javitch, J. A. Singlemolecule dynamics of gating in a neurotransmitter transporter homologue. Nature 2010, 465, 188-193.

(35) Cheng, M.; Bahar, I. Coupled Global and Local Changes Direct Substrate Translocation byNeurotransmitter-Sodium Symporter Ortholog LeuT. Biophys. J 2013, 105, 630-639.

(36) Cheng, M. H.; Bahar, I. Complete Mapping of Substrate Translocation Highlights the Role of LeuT N-terminal Segment in Regulating Transport Cycle. PLoS Comput. Biol. 2014, 10 .

(37) Andersen, J.; Stuhr-Hansen, N.; Zachariassen, L.; Toubro, S.; Hansen, S. M. R.; Eildal, J. N. N.; Bond, A. D.; Bges, K. P.; Bang-Andersen, B.; Kristensen, A. S.; Strmgaard, K. Molecular determinants for selective recognition of antidepressants in the human serotonin and norepinephrine transporters. Proc. Natl. Acad. Sci. U.S.A. 2011, $108,12137-12142$. 
(38) Mortensen, O. V.; Kortagere, S. Designing modulators of monoamine transporters using virtual screening techniques. Front Pharmacol 2015, 6 .

(39) Schlessinger, A.; Geier, E.; Fan, H.; Irwin, J. J.; Shoichet, B. K.; Giacomini, K. M.; Sali, A. Structure-based discovery of prescription drugs that interact with the norepinephrine transporter, NET. Proc. Natl. Acad. Sci. U.S.A. 2011, 108, 15810-15815.

(40) Cheng, M.; Bahar, I. Molecular Mechanism of Dopamine Transport by Human Dopamine Transporter. Structure 2015, 23, 2171-2181.

(41) Cheng, M. H.; Kaya, C.; Bahar, I. Quantitative Assessment of the Energetics of Dopamine Translocation by Human Dopamine Transporter. J. Phys. Chem. B 2018, 122, 5336-5346.

(42) Razavi, A. M.; Khelashvili, G.; Weinstein, H. A Markov State-based Quantitative Kinetic Model of Sodium Release from the Dopamine Transporter. Sci. Rep. 2017, 7, 40076.

(43) Cheng, M. H.; Bahar, I. Monoamine transporters: structure, intrinsic dynamics and allosteric regulation. Nat. Struct. Mol. Biol 2019, 26, 545-556.

(44) Coleman, J. A.; Green, E. M.; Gouaux, E. X-ray structures and mechanism of the human serotonin transporter. Nature 2016, 532, 334-339.

(45) Gabrielsen, M.; Ravna, A. W.; Kristiansen, K.; Sylte, I. Substrate binding and translocation of the serotonin transporter studied by docking and molecular dynamics simulations. J. Mol. Model. 2012, 18, 1073-1085.

(46) Penmatsa, A.; Wang, K. H.; Gouaux, E. X-ray structure of dopamine transporter elucidates antidepressant mechanism. Nature 2013, 503, 85-90.

(47) Xue, W.; Yang, F.; Wang, P.; Zheng, G.; Chen, Y.; Yao, X.; Zhu, F. What Contributes to SerotoninNorepinephrine Reuptake Inhibitors Dual-Targeting Mechanism? 
The Key Role of Transmembrane Domain 6 in Human Serotonin and Norepinephrine Transporters Revealed by Molecular Dynamics Simulation. ACS Chem. Neurosci. 2018, 9, 1128-1140.

(48) Wang, H.; Goehring, A.; Wang, K. H.; Penmatsa, A.; Ressler, R.; Gouaux, E. Structural basis for action by diverse antidepressants on biogenic amine transporters. Nature 2013, 503, 141-145.

(49) Coleman, J. A.; Gouaux, E. Structural basis for recognition of diverse antidepressants by the human serotonin transporter. Nat. Struct. Mol. Biol. 2018, 25, 170.

(50) Coleman, J. A.; Yang, D.; Zhao, Z.; Wen, P.-C.; Yoshioka, C.; Tajkhorshid, E.; Gouaux, E. Serotonin transporteribogaine complexes illuminate mechanisms of inhibition and transport. Nature 2019, 569, 141-145.

(51) Malinauskaite, L.; Quick, M.; Reinhard, L.; Lyons, J. A.; Yano, H.; Javitch, J. A.; Nissen, P. A mechanism for intracellular release of $\mathrm{Na}^{+}$by neurotransmitter/sodium symporters. Nat. Struct. Mol. Biol. 2014, 21, 1006-1012.

(52) Khelashvili, G.; Stanley, N.; Sahai, M. A.; Medina, J.; LeVine, M. V.; Shi, L.; De Fabritiis, G.; Weinstein, H. Spontaneous Inward Opening of the Dopamine Transporter Is Triggered by PIP2-Regulated Dynamics of the N-Terminus. ACS Chem. Neurosci. 2015, 6, 1825-1837.

(53) Kniazeff, J.; Shi, L.; Loland, C. J.; Javitch, J. A.; Weinstein, H.; Gether, U. An Intracellular Interaction Network Regulates Conformational Transitions in the Dopamine Transporter. J. Biol. Chem. 2008, 283, 17691-17701.

(54) Mller, I. R.; Slivacka, M.; Nielsen, A. K.; Rasmussen, S. G. F.; Gether, U.; Loland, C. J.; Rand, K. D. Conformational dynamics of the human serotonin transporter during substrate and drug binding. Nat. Commun. 2019, 10, 1687. 
(55) Nielsen, A. K.; Mller, I. R.; Wang, Y.; Rasmussen, S. G. F.; Lindorff-Larsen, K.; Rand, K. D.; Loland, C. J. Substrate-induced conformational dynamics of the dopamine transporter. Nat. Commun. 2019, 10, 2714.

(56) Merkle, P. S.; Gotfryd, K.; Cuendet, M. A.; Leth-Espensen, K. Z.; Gether, U.; Loland, C. J.; Rand, K. D. Substrate-modulated unwinding of transmembrane helices in the NSS transporter LeuT. Sci. Adv. 2018, 4, eaar6179.

(57) Selvam, B.; Mittal, S.; Shukla, D. Free Energy Landscape of the Complete Transport Cycle in a Key Bacterial Transporter. ACS Cent. Sci. 2018, 4, 1146-1154.

(58) Husic, B. E.; Pande, V. S. Markov State Models: From an Art to a Science. J. Am. Chem. Soc. 2018, 140, 2386-2396.

(59) Shukla, D.; Hernndez, C. X.; Weber, J. K.; Pande, V. S. Markov State Models Provide Insights into Dynamic Modulation of Protein Function. Acc. Chem. Res 2015, 48, $414-422$.

(60) Selvam, B.; Yu, Y.-C.; Chen, L.-Q.; Shukla, D. Molecular Basis of the Glucose Transport Mechanism in Plants. ACS Cent. Sci. 2019, 5, 1085-1096.

(61) Cheng, K. J.; Selvam, B.; Chen, L.-Q.; Shukla, D. Distinct Substrate Transport Mechanism Identified in Homologous Sugar Transporters. J. Phys. Chem. B 2019, 123, 8411-8418.

(62) Zhao, Y.; Terry, D. S.; Shi, L.; Quick, M.; Weinstein, H.; Blanchard, S. C.; Javitch, J. A. Substrate-modulated gating dynamics in a $\mathrm{Na}^{+}$-coupled neurotransmitter transporter homologue. Nature 2011, 474, 109-113.

(63) Quick, M.; Yano, H.; Goldberg, N. R.; Duan, L.; Beuming, T.; Shi, L.; Weinstein, H.; Javitch, J. A. State-dependent conformations of the translocation pathway in the ty- 
rosine transporter Tyt1, a novel neurotransmitter: sodium symporter from Fusobacterium nucleatum. J. Biol. Chem. 2006, 281, 26444-26454.

(64) Zhang, Y.-W.; Rudnick, G. The cytoplasmic substrate permeation pathway of serotonin transporter. J. Biol. Chem. 2006, 281, 36213-36220.

(65) Bjerregaard, H.; Severinsen, K.; Said, S.; Wiborg, O.; Sinning, S. A dualistic conformational response to substrate binding in the human serotonin transporter reveals a high affinity state for serotonin. J. Biol. Chem. 2015, 290, 7747-7755.

(66) Sun, P.; Li, J.; Zhang, X.; Guan, Z.; Xiao, Q.; Zhao, C.; Song, M.; Zhou, Y.; Mou, L.; Ke, M.; Guo, L.; Geng, J.; Deng, D. Crystal structure of the bacterial acetate transporter SatP reveals that it forms a hexameric channel. J. Biol. Chem. 2018, jbc.RA118.003876.

(67) Cao, Y. et al. Crystal structure of a potassium ion transporter, TrkH. Nature 2011, 471, 336-340.

(68) Machtens, J.-P.; Kortzak, D.; Lansche, C.; Leinenweber, A.; Kilian, P.; Begemann, B.; Zachariae, U.; Ewers, D.; deGroot, B.; Briones, R.; Fahlke, C. Mechanisms of Anion Conduction by Coupled Glutamate Transporters. Cell 2015, 160, 542-553.

(69) Campbell, N. G. et al. Structural, functional, and behavioral insights of dopamine dysfunction revealed by a deletion in SLC6A3. Proc. Natl. Acad. Sci. U.S.A. 2019, $116,3853-3862$.

(70) Billesbølle, C. B.; Mortensen, J. S.; Sohail, A.; Schmidt, S. G.; Shi, L.; Sitte, H. H.; Gether, U.; Loland, C. J. Transition metal ion FRET uncovers K+ regulation of a neurotransmitter/sodium symporter. Nat. Commun. 2016, 7, 12755.

(71) Nelson, P. J.; Rudnick, G. Coupling between platelet 5-hydroxytryptamine and potassium transport. J. Biol. Chem. 1979, 254, 10084-10089. 
(72) Benito-Muoz, C.; Perona, A.; Abia, D.; Dos Santos, H. G.; Nez, E.; Aragn, C.; LpezCorcuera, B. Modification of a Putative Third Sodium Site in the Glycine Transporter GlyT2 Influences the Chloride Dependence of Substrate Transport. Front. Mol. Neurosci $2018,11,347$.

(73) Subramanian, N.; Scopelitti, A. J.; Carland, J. E.; Ryan, R. M.; OMara, M. L.; Vandenberg, R. J. Identification of a 3rd Na+ Binding Site of the Glycine Transporter, GlyT2. PLoS One 2016, 11, e0157583.

(74) Korkhov, V. M.; Holy, M.; Freissmuth, M.; Sitte, H. H. The Conserved Glutamate (Glu136) in Transmembrane Domain 2 of the Serotonin Transporter Is Required for the Conformational Switch in the Transport Cycle. J. Biol. Chem. 2006, 281, 1343913448.

(75) Raley-Susman, K.; Cragoe, E.; Sapolsky, R.; Kopito, R. Regulation of intracellular $\mathrm{pH}$ in cultured hippocampal neurons by an amiloride-insensitive $\mathrm{Na}+/ \mathrm{H}+$ exchanger. J. Biol. Chem. 1991, 266, 2739-2745.

(76) Wang, K. H.; Penmatsa, A.; Gouaux, E. Neurotransmitter and psychostimulant recognition by the dopamine transporter. Nature 2015, 521, 322-327.

(77) Stolzenberg, S.; Li, Z.; Quick, M.; Malinauskaite, L.; Nissen, P.; Weinstein, H.; Javitch, J. A.; Shi, L. The role of transmembrane segment 5 (TM5) in Na2 release and the conformational transition of neurotransmitter:sodium symporters toward the inwardopen state. J. Biol. Chem. 2017, 292, 7372-7384.

(78) Koldsø, H.; Noer, P.; Grouleff, J.; Autzen, H. E.; Sinning, S.; Schiøtt, B. Unbiased simulations reveal the inward-facing conformation of the human serotonin transporter and $\mathrm{Na}+$ ion release. PLoS Comput. Biol. 2011, \%, e1002246.

(79) Khafizov, K.; Perez, C.; Koshy, C.; Quick, M.; Fendler, K.; Ziegler, C.; Forrest, L. R. 
Investigation of the sodium-binding sites in the sodium-coupled betaine transporter BetP. Proc. Natl. Acad. Sci. U.S.A. 2012, 109, E3035-E3044.

(80) Felts, B.; Pramod, A. B.; Sandtner, W.; Burbach, N.; Bulling, S.; Sitte, H. H.; Henry, L. K. The two $\mathrm{Na}+$ sites in the human serotonin transporter play distinct roles in the ion coupling and electrogenicity of transport. J. Biol. Chem. 2014, 289, $1825-1840$.

(81) Andersen, J.; Stuhr-Hansen, N.; Zachariassen, L. G.; Kolds, H.; Schitt, B.; Strmgaard, K.; Kristensen, A. S. Molecular Basis for Selective Serotonin Reuptake Inhibition by the Antidepressant Agent Fluoxetine (Prozac). Mol. Pharmacol. 2014, 85, 703-714.

(82) Srensen, L.; Andersen, J.; Thomsen, M.; Hansen, S. M. R.; Zhao, X.; Sandelin, A.; Strmgaard, K.; Kristensen, A. S. Interaction of antidepressants with the serotonin and norepinephrine transporters: mutational studies of the S1 substrate binding pocket. J. Biol. Chem. 2012, 287, 43694-43707.

(83) Barker, E. L.; Moore, K. R.; Rakhshan, F.; Blakely, R. D. Transmembrane domain I contributes to the permeation pathway for serotonin and ions in the serotonin transporter. J. Neurosci. 1999, 19, 4705-4717.

(84) Celik, L.; Sinning, S.; Severinsen, K.; Hansen, C. G.; Møller, M. S.; Bols, M.; Wiborg, O.; Schiøtt, B. Binding of serotonin to the human serotonin transporter. Molecular modeling and experimental validation. J. Am. Chem. Soc. 2008, 130, 3853-3865.

(85) Andersen, J.; Olsen, L.; Hansen, K. B.; Taboureau, O.; Jrgensen, F. S.; Jrgensen, A. M.; Bang-Andersen, B.; Egebjerg, J.; Strmgaard, K.; Kristensen, A. S. Mutational mapping and modeling of the binding site for (S)-citalopram in the human serotonin transporter. J. Biol. Chem. 2010, 285, 2051-2063. 
(86) Kolds, H.; Severinsen, K.; Tran, T. T.; Celik, L.; Jensen, H. H.; Wiborg, O.; Schitt, B.; Sinning, S. The two enantiomers of citalopram bind to the human serotonin transporter in reversed orientations. J. Am. Chem. Soc. 2010, 132, 1311-1322.

(87) Barker, E. L.; Perlman, M. A.; Adkins, E. M.; Houlihan, W. J.; Pristupa, Z. B.; Niznik, H. B.; Blakely, R. D. High Affinity Recognition of Serotonin Transporter Antagonists Defined By Species-Scanning Mutagenesis An Aromatic Residue in Transmembrane Domain I Dictates Species-Selective Recognition of Citalopram and Mazindol. J. Biol. Chem. 1998, 273, 19459-19468.

(88) Hellsberg, E.; Ecker, G. F.; Stary-Weinzinger, A.; Forrest, L. R. A structural model of the human serotonin transporter in an outward-occluded state. PLoS One 2019, $14, \mathrm{e} 0217377$.

(89) Barker, E. L.; Blakely, R. D. Methods Enzymol; Elsevier, 1998; Vol. 296; pp 475-498.

(90) Solis, E.; Zdravkovic, I.; Tomlinson, I. D.; Noskov, S. Y.; Rosenthal, S. J.; De Felice, L. J. 4-(4-(dimethylamino) phenyl)-1-methylpyridinium $(\mathrm{APP}+)$ is a fluorescent substrate for the human serotonin transporter. J. Biol. Chem. 2012, 287, 8852-8863.

(91) Zeppelin, T.; Ladefoged, L. K.; Sinning, S.; Periole, X.; Schiøtt, B. A direct interaction of cholesterol with the dopamine transporter prevents its out-to-inward transition. PLoS Comput. Biol. 2018, 14, e1005907.

(92) Laursen, L.; Severinsen, K.; Kristensen, K. B.; Periole, X.; Overby, M.; Müller, H. K.; Schiøtt, B.; Sinning, S. Cholesterol binding to a conserved site modulates the conformation, pharmacology, and transport kinetics of the human serotonin transporter. $J$. Biol. Chem. 2018, 293, 3510-3523.

(93) Anderluh, A.; Klotzsch, E.; Reismann, A. W. A. F.; Brameshuber, M.; Kudlacek, O.; Newman, A. H.; Sitte, H. H.; Schtz, G. J. Single molecule analysis reveals coexis- 
tence of stable serotonin transporter monomers and oligomers in the live cell plasma membrane. J. Biol. Chem. 2014, 289, 4387-4394.

(94) Anderluh, A.; Hofmaier, T.; Klotzsch, E.; Kudlacek, O.; Stockner, T.; Sitte, H. H.; Schtz, G. J. Direct $\mathrm{PIP}_{2}$ binding mediates stable oligomer formation of the serotonin transporter. Nat. Commun. 2017, 8, 14089.

(95) Periole, X.; Zeppelin, T.; Schitt, B. Dimer Interface of the Human Serotonin Transporter and Effect of the Membrane Composition. Sci. Rep. 2018, 8, 5080.

(96) Jo, S.; Kim, T.; Iyer, V. G.; Im, W. CHARMM-GUI: a web-based graphical user interface for CHARMM. J. Comput. Chem. 2008, 29, 1859-1865.

(97) Jorgensen, W. L.; Chandrasekhar, J.; Madura, J. D.; Impey, R. W.; Klein, M. L. Comparison of simple potential functions for simulating liquid water. J. Chem. Phys. 1983, 79, 926-935.

(98) Case, D. A. et al. Amber 2014. 2014,

(99) Gotz, A. W.; Williamson, M. J.; Xu, D.; Poole, D.; Le Grand, S.; Walker, R. C. Routine microsecond molecular dynamics simulations with AMBER on GPUs. 1. Generalized born. J. Chem. Theory Comput. 2012, 8, 1542-1555.

(100) Salomon-Ferrer, R.; Gotz, A. W.; Poole, D.; Le Grand, S.; Walker, R. C. Routine microsecond molecular dynamics simulations with AMBER on GPUs. 2. Explicit solvent particle mesh Ewald. J. Chem. Theory Comput. 2013, 9, 3878-3888.

(101) Le Grand, S.; Götz, A. W.; Walker, R. C. SPFP: Speed without compromiseA mixed precision model for GPU accelerated molecular dynamics simulations. Comput. Phys. Commun. 2013, 184, 374-380. 
(102) Maier, J. A.; Martinez, C.; Kasavajhala, K.; Wickstrom, L.; Hauser, K. E.; Simmerling, C. ff14SB: Improving the Accuracy of Protein Side Chain and Backbone Parameters from ff99SB. J. Chem. Theory Comput. 2015, 11, 3696-3713.

(103) Berendsen, H. J. C.; Postma, J. P. M.; van Gunsteren, W. F.; DiNola, A.; Haak, J. R. Molecular dynamics with coupling to an external bath. J. Chem. Phys. 1984, 81, 3684-3690.

(104) York, D. M.; Darden, T. A.; Pedersen, L. G. The effect of longrange electrostatic interactions in simulations of macromolecular crystals: A comparison of the Ewald and truncated list methods. J. Chem. Phys. 1993, 99, 8345-8348.

(105) Krutler, V.; Gunsteren, W. F. v.; Hnenberger, P. H. A fast SHAKE algorithm to solve distance constraint equations for small molecules in molecular dynamics simulations. J. Comput. Chem. 2001, 22, 501-508.

(106) Bowman, G. R.; Ensign, D. L.; Pande, V. S. Enhanced Modeling via Network Theory: Adaptive Sampling of Markov State Models. J. Chem. Theory Comput. 2010, 6, 787794.

(107) Kohlhoff, K. J.; Shukla, D.; Lawrenz, M.; Bowman, G. R.; Konerding, D. E.; Belov, D.; Altman, R. B.; Pande, V. S. Cloud-based simulations on Google Exacycle reveal ligand modulation of GPCR activation pathways. Nat. Chem. 2014, 6, 15-21.

(108) Sculley, D. Web-scale k-means clustering. Proceedings of the 19th international conference on World wide web - WWW'10. Raleigh, North Carolina, USA, 2010; p 1177.

(109) Roe, D. R.; Cheatham, T. E. PTRAJ and CPPTRAJ: Software for Processing and Analysis of Molecular Dynamics Trajectory Data. J. Chem. Theory Comput. 2013, 9, 3084-3095. 
(110) McGibbon, R.; Beauchamp, K.; Harrigan, M.; Klein, C.; Swails, J.; Hernndez, C.; Schwantes, C.; Wang, L.-P.; Lane, T.; Pande, V. MDTraj: A Modern Open Library for the Analysis of Molecular Dynamics Trajectories. Biophys. J 2015, 109, 15281532.

(111) Scherer, M. K.; Trendelkamp-Schroer, B.; Paul, F.; Prez-Hernndez, G.; Hoffmann, M.; Plattner, N.; Wehmeyer, C.; Prinz, J.-H.; No, F. PyEMMA 2: A Software Package for Estimation, Validation, and Analysis of Markov Models. J. Chem. Theory Comput. 2015, 11, 5525-5542.

(112) Naritomi, Y.; Fuchigami, S. Slow dynamics in protein fluctuations revealed by timestructure based independent component analysis: the case of domain motions. J. Chem. Phys. 2011, 134, 02B617.

(113) Prinz, J.-H.; Wu, H.; Sarich, M.; Keller, B.; Senne, M.; Held, M.; Chodera, J. D.; Schütte, C.; Noé, F. Markov models of molecular kinetics: Generation and validation. J. Chem. Phys. 2011, 134, 174105.

(114) Humphrey, W.; Dalke, A.; Schulten, K. VMD: Visual molecular dynamics. J. Mol. Graph. 1996, 14, 33-38.

(115) Smart, O. S.; Neduvelil, J. G.; Wang, X.; Wallace, B. A.; Sansom, M. S. P. HOLE: A program for the analysis of the pore dimensions of ion channel structural models. $J$. Mol. Graph. 1996, 14, 354-360.

(116) Pahari, S.; Sun, L.; Basu, S.; Alexov, E. DelPhiPKa: Including salt in the calculations and enabling polar residues to titrate. Proteins 2018, 86, 1277-1283.

(117) Metzner, P.; Schtte, C.; Vanden-Eijnden, E. Transition Path Theory for Markov Jump Processes. Multiscale Model Sim. 2009, 7, 1192-1219. 
(118) No, F.; Schtte, C.; Vanden-Eijnden, E.; Reich, L.; Weikl, T. R. Constructing the equilibrium ensemble of folding pathways from short off-equilibrium simulations. Proc. Natl. Acad. Sci. U.S.A. 2009, 106, 19011-19016.

(119) Case, D. A. et al. AMBER 2018. University of California, San Francisco 2018,

(120) Grossfield, A. WHAM: the weighted histogram analysis method, version 2.0. 9. Available at membrane. urmc. rochester. edu/content/wham. Accessed November 2013, 15, 2013.

(121) Ramamoorthy, S.; Bauman, A. L.; Moore, K. R.; Han, H.; Yang-Feng, T.; Chang, A. S.; Ganapathy, V.; Blakely, R. D. Antidepressant-and cocaine-sensitive human serotonin transporter: molecular cloning, expression, and chromosomal localization. Proc. Natl. Acad. Sci. U.S.A. 1993, 90, 2542-2546.

(122) Morris, G. M.; Huey, R.; Lindstrom, W.; Sanner, M. F.; Belew, R. K.; Goodsell, D. S.; Olson, A. J. AutoDock4 and AutoDockTools4: Automated docking with selective receptor flexibility. J. Comput. Chem. 2009, 30, 2785-2791.

(123) Morris, G. M.; Goodsell, D. S.; Halliday, R. S.; Huey, R.; Hart, W. E.; Belew, R. K.; Olson, A. J. Automated docking using a Lamarckian genetic algorithm and an empirical binding free energy function. J. Comput. Chem. 1998, 19, 1639-1662. 
Graphical TOC Entry

1008

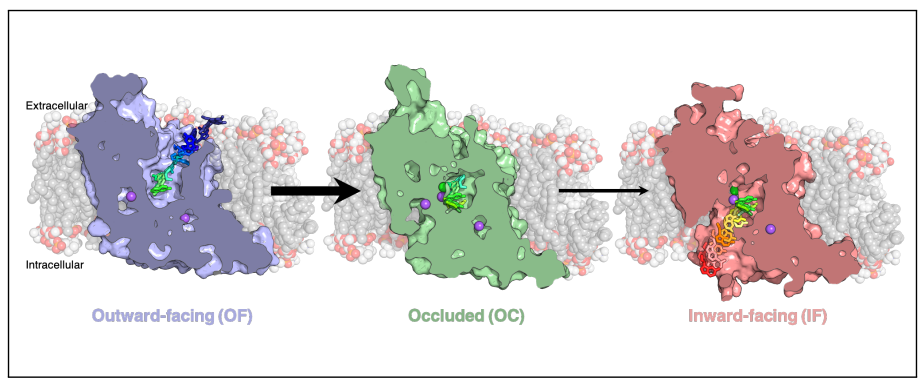

